SI-HEP-2006-03

\title{
Form Factors from Light-Cone Sum Rules with $B$-Meson Distribution Amplitudes
}

\author{
Alexander Khodjamirian, Thomas Mannel and Nils Offen \\ Theoretische Physik 1, Fachbereich Physik, Universität Siegen, \\ D-57068 Siegen, Germany
}

\begin{abstract}
New sum rules for $B \rightarrow \pi, K$ and $B \rightarrow \rho, K^{*}$ form factors are derived from the correlation functions expanded near the light-cone in terms of $B$-meson distribution amplitudes. The contributions of quark-antiquark and quark-antiquark-gluon components in the $B$ meson are taken into account. Models for the $B$-meson three-particle distribution amplitudes are suggested, based on QCD sum rules in HQET. Employing the new light-cone sum rules we calculate the form factors at small momentum transfers, including $S U(3)$-violation effects. The results agree with the predictions of the conventional light-cone sum rules.
\end{abstract}




\section{Introduction}

There is a growing demand for more accurate and reliable calculations of heavyto-light transition form factors in QCD. The $B \rightarrow P, V$ form factors with $P=\pi, K$ and $V=\rho, K^{*}$ final states provide the hadronic input in exclusive semileptonic $B \rightarrow P(V) l \nu_{l}, \quad B \rightarrow P(V) \bar{l} l$ and radiative $B \rightarrow V \gamma$ decays. The same form factors determine factorizable amplitudes in the nonleptonic charmless $B$-decays. All these decay channels are used for determination of CKM parameters and for various tests of Standard Model. Pinning down the uncertainty of the form factors is in many cases the only way to increase the precision of these analyses.

Lattice QCD is successfully used to calculate heavy-to-light form factors in the region of large momentum transfer squared, $q^{2}=\left(p_{B}-p_{P, V}\right)^{2}$. To access small $q^{2}$ (large energies of the light hadron), other QCD-based approaches are employed, such as the light-cone sum rules (LCSR) [1] for $B \rightarrow P$ [2, 3] and $B \rightarrow V$ [4, 5] form factors. To derive a LCSR, one starts with the operatorproduct expansion (OPE) of a dedicated correlation function near the light-cone. The OPE result is then combined with the hadronic dispersion relation and quark-hadron duality, hence there are many common features with the original QCD sum rules [6]. In the standard LCSR approach (hereafter called lightmeson LCSR), the correlation function is taken between the vacuum and light $P$ - or $V$-meson state, whereas the $B$ meson is interpolated by a heavy-light quark current. As a result, the long-distance dynamics in the correlation function is described by a set of pion, kaon, or $\rho$-, $K^{*}$-meson distribution amplitudes (DA's) of low twists. The main uncertainties in the light-meson LCSR originate from the limited accuracy of the DA parameters. In addition, a sort of "systematic" uncertainty is brought by the quark-hadron duality approximation in the $B$ meson channel. Hence, it is desirable to confirm the predictions of the lightmeson LCSR by calculating the same form factors in an independent way, using different input and assumptions.

A different sum rule for the $B \rightarrow \pi$ form factor was recently suggested by us in [7] and, independently, in the framework of SCET in [8]. The main idea is to "invert " the correlation function, that is, to interpolate the pion with an appropriate light-quark (axial-vector) current, and put the $B$ meson on-shell using the light-cone expansion in terms of the $B$-meson DA's. The latter are universal nonperturbative objects introduced in the framework of HQET [9] (see also [10; a review can be found in [11]) and used in several factorization formulae for exclusive $B$-decays (see e.g., [12, 13, 14, 15]).

In this paper the new version of LCSR (we call it B-meson LCSR) is developed further. Following [7, we introduce the $B$-to-vacuum correlation function and prove its light-cone dominance. The new $B$-meson LCSR for several phenomenologically important $B \rightarrow P, V$ form factors at $q^{2} \geq 0$ are derived. In addition to the leading-order contributions of the two-particle (quark-antiquark) $B$-meson DA's $\phi_{+}^{B}$ and $\phi_{-}^{B}$, we calculate the corrections due to the three-particle (quark-antiquark-gluon) DA's defined in [16. The functional form of the latter DA's was not known previously. Following [9, 17], we derive additional QCD sum rules for the vacuum correlation function of two heavy-light currents in HQET. We then use the perturbative parts of these sum rules to fix the behavior of the three-particle $B$-meson DA's at small light-cone momenta of the spectator quark and gluon. First models for three-particle DA's are suggested in 
which the "infrared" behavior obtained from the HQET sum rules is combined with the large-momentum fall-off. We find that the simple exponential ansatz for the two-particle DA's suggested in [9] and the exponential version of our model for the three-particle DA's form a selfconsistent set, so that the relations between $B$-meson DA's [16] following from the equations of motion are fulfilled.

Our model, as well as LCSR obtained below, do not include QCD radiative corrections which are beyond the scope of this work. NLO effects have already been taken into account in more elaborated models of $\phi_{+}^{B}$ based on HQET sum rules [17, or on the first two moments [18. The most important effect in NLO is the "radiative tail" of $\phi_{+}^{B}(\omega)$ at $\omega \rightarrow \infty$ caused by the nontrivial renormalization [19] of the effective heavy-light current. Importantly, this peculiar ultraviolet behavior of $B$-meson DA's plays no role in LCSR at the leading, $O\left(\alpha_{s}^{0}\right)$ order, where a sort of an end-point mechanism dominates. The duality threshold in the sum rule cuts off the integration over the spectator momentum $\omega$ well below the region where the effect of the tail becomes noticeable. Note that radiative corrections to the LCSR in SCET for the $B \rightarrow \pi$ form factor have already been calculated in [8], and their numerical impact is moderate.

The LCSR obtained in this paper depend on the parameters determining the $B$-meson DA's. The most important input is $\lambda_{B}$, the first inverse moment of $\phi_{+}^{B}(\omega)$. At the same time, the new sum rules are independent of the DA's of $\pi, K$ or $\rho, K^{*}$ mesons. The light mesons are now interpolated by the lightquark currents, hence the new sum rules rely on the quark-hadron duality in the channels of these currents. The duality-threshold parameter in each channel is determined from the corresponding two-point QCD (SVZ) sum rule for the light-meson decay constant. Furthermore, the $S U(3)$-violation effects are calculated in terms of the $s$-quark mass and the differences in the duality-threshold parameters for strange and nonstrange mesons.

From the new sum rules we obtain numerical predictions for various $B \rightarrow$ $\pi, K$ and $B \rightarrow \rho, K^{*}$ form factors. Our main observation is the sensitivity of the form factors to the input value of $\lambda_{B}$. This circumstance was already used in 7 ] to extract the interval for $\lambda_{B}$ using the light-meson LCSR result for the $B \rightarrow \pi$ form factor $f_{B \pi}^{+}$. Here, in order to be independent of the light-meson LCSR, we use the interval of $\lambda_{B}$ inferred from QCD sum rules in HQET [17. With this input, we observe a good agreement of the predicted form factors with the most recent results from the light-meson LCSR obtained in [3, 5.

In what follows, in section 2 we introduce the correlation function and discuss the applicability of the light-cone expansion. The sum rules in the leading order including the contributions of two- and three-particle DA's are derived in section 3 . In section 4 , the $B$-meson three-particle DA's are investigated and their form at small momenta of light-quark and gluon is established. The models of threeparticle DA's are suggested and the relations between two- and three-particle DA's following from the equations of motion are investigated. In section 5 we discuss the heavy-mass dependence of the form factors obtained from $B$-meson LCSR. Section 6 contains the numerical results for the form factors and the concluding discussion. In the Appendix we present the bulky expressions for the sum rules at nonvanishing light-quark mass and nonzero momentum transfer. 


\begin{tabular}{|c|c|c|c|c|c|}
\hline Transition & $q_{1}$ & $q_{2}$ & $\Gamma_{a}$ & $\Gamma_{b}$ & Form factors \\
\hline \hline $\bar{B} \rightarrow \pi$ & $u$ & $d, u$ & $\gamma_{\nu} \gamma_{5}$ & $\gamma_{\mu}$ & $f_{B \pi}^{+}, f_{B \pi}^{-}$ \\
\hline $\bar{B} \rightarrow K$ & $s$ & $d, u$ & $\gamma_{\nu} \gamma_{5}$ & $\gamma_{\mu}$ & $f_{B \pi}^{T}$ \\
\hline $\bar{B} \rightarrow \rho$ & $u$ & $d . u$ & $\gamma_{\nu}, f_{B K}^{-}$ & $f_{B K}^{T} \gamma_{5}$ & $A_{1}^{B \rho}, A_{2}^{B \rho}$ \\
\hline $\bar{B} \rightarrow K^{*}$ & $s$ & $d, u$ & $\gamma_{\nu}$ & $\gamma_{\mu} \gamma_{5}$ & $A_{1}^{B K^{*}}, A_{2}^{B K^{*}}$ \\
& & & & $\sigma_{\mu \rho}$ & $T_{1}^{B \rho}$ \\
\hline & & & & $\sigma_{\mu \rho}$ & $T_{1}^{B K^{*}}$ \\
\hline
\end{tabular}

Table 1: Combinations of light-quark flavours and Dirac matrices in the correlation function (1) and the corresponding heavy-to-light form factors.

\section{Correlation function}

Following [7, we define a generic correlation function of two quark currents sandwiched between the vacuum and the on-shell $\bar{B}$-meson state:

$$
F_{a b}^{(B)}(p, q)=i \int d^{4} x e^{i p \cdot x}\left\langle 0\left|T\left\{\bar{q}_{2}(x) \Gamma_{a} q_{1}(x), \bar{q}_{1}(0) \Gamma_{b} b(0)\right\}\right| \bar{B}\left(P_{B}\right)\right\rangle,
$$

where $\bar{q}_{1} \Gamma_{b} b$ is one of the heavy-light (electro)weak currents and $\bar{q}_{2} \Gamma_{a} q_{1}$ is the interpolating current for a pseudoscalar or vector meson, with the flavour content determined by the valence quarks $q_{1,2}$. The external momenta of the currents are $q$ and $p$ respectively, and $P_{B}^{2}=(p+q)^{2}=m_{B}^{2}$. In Table 1 we list the combinations of quark flavours $q_{1}, q_{2}$ and Dirac-matrices $\Gamma_{a, b}$ for all $\bar{B} \rightarrow P, V$ transitions and their form factors considered in this paper. According to our choice, light pseudoscalar (vector) mesons are interpolated with the axial-vector (longitudinal vector) currents.

First of all, we have to convince ourselves that OPE near the light-cone is applicable for the correlation function (11) if the variables $p^{2}$ and $q^{2}$ are far below the hadronic thresholds in the channels of $\overline{q_{2}} \Gamma_{a} q_{1}$ and $\overline{q_{1}} \Gamma_{b} b$ currents, respectively. The correlation function can be systematically expanded in the 
limit of large $m_{b}$ in HQET. Separating the static momentum of the $B$-meson state, we rewrite $P_{B}=p+q=m_{b} v+k$, where $v$ is the four-velocity of $B$, and $k$ is the residual momentum. We retain the relativistic normalization of the state: $\left|B\left(P_{B}\right)\right\rangle=\left|B_{v}\right\rangle$, up to $1 / m_{b}$ corrections. Also the $b$-quark field is substituted by the effective field, using $b(x)=e^{-i m_{b} v x} h_{v}(x)$. For simplicity we consider the rest frame $v=(1,0,0,0)$. In first approximation, $m_{B}=m_{b}+\bar{\Lambda}$, hence $k_{0} \sim \bar{\Lambda}$ in this frame. We also redefine the four-momentum transfer $q$ by separating the "static" part of it: $q=m_{b} v+\tilde{q}$, so that $p+\tilde{q}=k$. After the transition to HQET,

$$
F_{a b}^{(B)}(p, q)=\tilde{F}_{a b}^{\left(B_{v}\right)}(p, \tilde{q})+O\left(1 / m_{b}\right),
$$

the correlation function in the heavy $m_{b}$ limit,

$$
\tilde{F}_{a b}^{\left(B_{v}\right)}(p, \tilde{q})=i \int d^{4} x e^{i p \cdot x}\left\langle 0\left|T\left\{\bar{q}_{2}(x) \Gamma_{a} q_{1}(x), \bar{q}_{1}(0) \Gamma_{b} h_{v}(0)\right\}\right| \bar{B}_{v}\right\rangle
$$

does not depend on $m_{b}$, if $p^{2}$ and $\tilde{q}^{2}$ are generic scales. In this amplitude two light-quark currents (one of them containing the effective field $h_{v}$ ) with virtualities $p^{2}$ and $\tilde{q}^{2}$ annihilate an effective hadronic state with a mass of $O(\bar{\Lambda})$. From the QCD point of view, the correlation function (3) resembles the $\gamma^{*}(p) \gamma^{*}(\tilde{q}) \rightarrow \pi^{0}(p+\tilde{q})$ transition amplitude. For the latter a detailed proof of the light-cone dominance can be found, e.g., in [20. Following the same line

of arguments for the amplitude $\tilde{F}_{a b}^{\left(B_{v}\right)}(p, \tilde{q})$, we assume that both four-momenta are spacelike, $p^{2}, \tilde{q}^{2}<0$, and sufficiently large:

$$
P^{2},\left|\tilde{q}^{2}\right| \gg \Lambda_{Q C D}^{2}, \bar{\Lambda}^{2},
$$

where $P^{2} \equiv-p^{2}$. Simultaneously, the difference between the virtualities is kept large, so that the ratio

$$
\xi=\frac{2 p \cdot k}{P^{2}} \sim \frac{\left|\tilde{q}^{2}\right|-P^{2}}{P^{2}} \neq 0,
$$

is at least of $O(1)$. With these conditions fulfilled, the integral in (3) is supported in the region of small $x^{2} \leq 1 / P^{2}$, where the exponent $e^{i p x}$ does not oscillate strongly.

Returning to the momentum-transfer squared $q^{2}$, one obtains

$$
q^{2} \simeq m_{b}^{2}+2 m_{b} \tilde{q}_{0} \sim m_{b}^{2}-m_{b} P^{2} \xi / \bar{\Lambda} .
$$

Thus, $q^{2}$ is far from the threshold $\sim m_{b}^{2}$ in the heavy-light channel, if the conditions (44) and (5) are fulfilled. Parametrically, the lower part of the physical region of $B \rightarrow P, V$ transitions

$$
0 \leq q^{2}<m_{b}^{2}-m_{b} P^{2} / \bar{\Lambda}
$$

is accessible to OPE on the light-cone. One encounters a situation similar to the light-meson LCSR which are applicable up to $q^{2}=m_{b}^{2}-m_{b} \chi$, where $\chi=O(1 \mathrm{GeV})$ does not scale with $m_{b} \rightarrow \infty$. For example, the LCSR with pion DA's [2] can be used up to $q^{2} \leq 14-16 \mathrm{GeV}^{2}$. For the $B$-meson LCSR considered here, the upper limit of the interval (7) could not be that large, because generally $P^{2} / \bar{\Lambda} \gg \chi$. An important case is when $q^{2}$ is in the vicinity of zero. Solving 


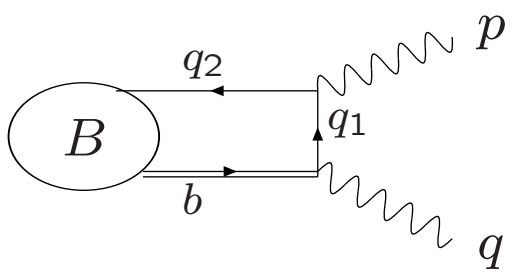

(a)

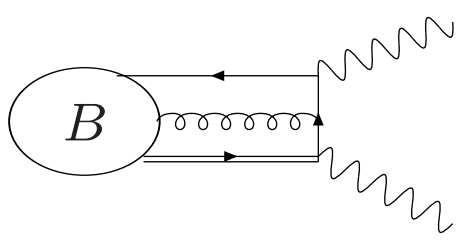

(b)

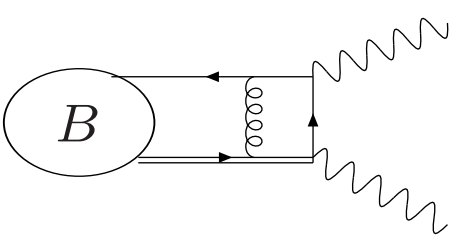

(c)

Figure 1: Diagrams corresponding to the contributions of (a) two-particle and (b) three-particle $B$-meson DA's to the correlation function (1); (c)-one of the $O\left(\alpha_{s}\right)$ diagrams. Curly (wavy) lines denote gluons (external currents).

Eq. (6) for $q^{2}=0$ one obtains $P^{2} \xi \sim m_{b} \bar{\Lambda}$. With $P^{2}$ being large but independent of $m_{b}$, in this case $\tilde{q}^{2}$ scales with $m_{b} \rightarrow \infty:\left|\tilde{q}^{2}\right|=P^{2}(1+\xi) \sim m_{b} \bar{\Lambda}$.

The light-cone dominance of the correlation function allows one to contract the $q_{1}$ and $\bar{q}_{1}$ fields and use the free-quark propagator $S_{q_{1}}(x)=-i\left\langle 0\left|q_{1}(x) \bar{q}_{1}(0)\right| 0\right\rangle$ as a leading-order approximation. The corresponding diagram is depicted in Fig. 1a. We obtain from Eq. (3) (neglecting for simplicity the light-quark mass $\left.m_{q_{1}}\right)$ :

$$
\tilde{F}_{a b}^{\left(B_{v}\right)}(p, \tilde{q})=i \int d^{4} x e^{i p \cdot x} \frac{i x_{\rho}}{2 \pi^{2}\left(x^{2}\right)^{2}}\left[\Gamma_{a} \gamma_{\rho} \Gamma_{b}\right]_{\alpha \beta}\left\langle 0\left|\bar{q}_{2 \alpha}(x) h_{v \beta}(0)\right| \bar{B}_{v}\right\rangle,
$$

a convolution of a short-distance part with the matrix element of the bilocal operator between the vacuum and $B_{v}$-state. Expanding the operator $\bar{q}_{2 \alpha}(x) h_{v \beta}(0)$ at $x=0$ one encounters, in the generic case $\xi \sim 1$, an infinite series of matrix elements of local operators, as explained in details in 21, 20, for the vacuumpion amplitudes. Instead, one has to retain in Eq. (8) the matrix element of the bilocal operator, expanding it around $x^{2}=0$. This procedure brings the $B$-meson DA's into the game. They however do not have a well defined twist, contrary to the light-meson DA's. The definitions of two- and three-particle $B$-meson DA's at the leading order of $x^{2} \rightarrow 0$ expansion will be given in the next section. 


\section{Derivation of LCSR}

The sum rules are obtained following the standard procedure [6], that is, matching the OPE result for the correlation function to the hadronic representation and employing quark-hadron duality and Borel transformation. Considering, for definiteness, the case when the current $\bar{q}_{2} \Gamma_{a} q_{1}$ interpolates a pseudoscalar meson $P(\pi$ or $K)$, we write the correlation function (11) in a form of the hadronic dispersion relation in the channel of the light meson:

$$
F_{a b}^{(B)}(p, q)=\frac{\left\langle 0\left|\bar{q}_{2} \Gamma_{a} q_{1}\right| P(p)\right\rangle\left\langle P(p)\left|\bar{q}_{1} \Gamma_{b} b\right| \bar{B}\left(P_{B}\right)\right\rangle}{m_{P}^{2}-p^{2}}+\ldots,
$$

where only the $P$-meson pole term is shown explicitly, and ellipses indicate the contributions of excited and continuum states. The two hadronic matrix elements in Eq. (9) are determined, respectively, by the decay constant of $P$ and by the $B \rightarrow P$ form factors.

To proceed, the dispersion relation (9) is equated to the HQET correlation function (8), which will be calculated using light-cone OPE:

$$
F_{a b}^{(B), O P E}(p, q) \simeq \tilde{F}_{a b}^{\left(B_{v}\right), O P E}\left(p, q-m_{b} v\right) .
$$

After Lorentz-decomposition,

$$
F_{a b}^{(B), O P E}(p, q)=l_{a b}(p, q) F^{(B), O P E}\left(p^{2}, q^{2}\right)+\ldots,
$$

each invariant amplitude is conveniently represented in a form of dispersion relation:

$$
F^{(B), O P E}\left(p^{2}, q^{2}\right)=\frac{1}{\pi} \int_{\left(m_{q_{1}}+m_{q_{2}}\right)^{2}}^{\infty} d s \frac{\operatorname{Im} F^{(B), O P E}\left(s, q^{2}\right)}{s-p^{2}},
$$

where the lower threshold is given by the sum of the light-quark masses. Furthermore, employing quark-hadron duality approximation we equate the P-meson contribution in Eq. (9) to the part of the dispersion integral (11) limited from above by the effective threshold $s_{0}^{P}$. After Borel transformation, the LCSR for the $B \rightarrow P$ matrix element can be written down in the following generic form:

$$
\begin{array}{r}
\left\langle 0\left|\bar{q}_{2} \Gamma_{a} q_{1}\right| P(p)\right\rangle\left\langle P(p)\left|\bar{q}_{1} \Gamma_{b} b\right| \bar{B}\left(P_{B}\right)\right\rangle e^{-m_{P}^{2} / M^{2}} \\
=l_{a b}(p, q) \int_{\left(m_{q_{1}}+m_{q_{2}}\right)^{2}}^{s_{0}^{P}} d s e^{-s / M^{2}} \operatorname{Im} F^{(B), O P E}\left(s, q^{2}\right)+\ldots,
\end{array}
$$

where the ellipses denote the rest of the Lorentz-decomposition. The derivation of LCSR in the case of a vector meson $V\left(\rho\right.$ or $\left.K^{*}\right)$ is fully analogous, with $V$ replacing $P$ in Eqs. (9), (12). For each combination of currents listed in Table 1, using the definitions of the hadronic matrix elements given below and decomposing the l.h.s of Eq. (12) in invariant amplitudes it is straightforward to obtain a separate sum rule for a given form factor.

The following standard definitions are used for the decay constants of pseudoscalar and vector mesons:

$$
\begin{aligned}
& \kappa\left\langle 0\left|\bar{q}_{2} \gamma_{\nu} \gamma_{5} q_{1}\right| P(p)\right\rangle=i p_{\nu} f_{P}, \\
& \kappa\left\langle 0\left|\bar{q}_{2} \gamma_{\nu} q_{1}\right| V(p)\right\rangle=\epsilon_{\nu}^{V} m_{V} f_{V},
\end{aligned}
$$


for $B \rightarrow P$ form factors:

$$
\begin{aligned}
& \kappa\left\langle P(p)\left|\overline{q_{1}} \gamma_{\mu} b\right| \bar{B}(p+q)\right\rangle=2 p_{\mu} f_{B P}^{+}\left(q^{2}\right)+q_{\mu}\left[f_{B P}^{+}\left(q^{2}\right)+f_{B P}^{-}\left(q^{2}\right)\right] \\
& \kappa\left\langle P(p)\left|\overline{q_{1}} \sigma_{\mu \rho} q^{\rho} b\right| B(p+q)\right\rangle=\left[q^{2}\left(2 p_{\mu}+q_{\mu}\right)-\left(m_{B}^{2}-m_{P}^{2}\right) q_{\mu}\right] \frac{i f_{B P}^{T}\left(q^{2}\right)}{m_{B}+m_{P}},
\end{aligned}
$$

and for $B \rightarrow V$ form factors:

$$
\begin{array}{r}
\kappa\left\langle V(p)\left|\overline{q_{1}} \gamma_{\mu}\left(1-\gamma_{5}\right) b\right| \bar{B}(p+q)\right\rangle=-i \epsilon_{\mu}^{*}\left(m_{B}+m_{V}\right) A_{1}^{B V}\left(q^{2}\right) \\
+i(2 p+q)_{\mu}\left(\epsilon^{*} q\right) \frac{A_{2}^{B V}\left(q^{2}\right)}{m_{B}+m_{V}}+i q_{\mu}\left(\epsilon^{*} q\right) \frac{2 m_{V}}{q^{2}}\left(A_{3}^{B V}\left(q^{2}\right)-A_{0}^{B V}\left(q^{2}\right)\right) \\
+\epsilon_{\mu \nu \rho \sigma} \epsilon^{* \nu} q^{\rho} p^{\sigma} \frac{2 V^{B V}\left(q^{2}\right)}{m_{B}+m_{V}},
\end{array}
$$

with $2 m_{V} A_{3}^{B V}\left(q^{2}\right)=\left(m_{B}+m_{V}\right) A_{1}\left(q^{2}\right)-\left(m_{B}-m_{V}\right) A_{2}^{B V}\left(q^{2}\right)$ and $A_{0}^{B V}(0)=$ $A_{3}^{B V}(0)$, and

$$
\begin{aligned}
\kappa\langle V(p)| \overline{q_{1}} \sigma_{\mu \rho} q^{\rho}(1 & \left.+\gamma_{5}\right) b|\bar{B}(p+q)\rangle=i \epsilon_{\mu \nu \rho \sigma} \epsilon^{* \nu} q^{\rho} p^{\sigma} 2 T_{1}^{B V}\left(q^{2}\right) \\
& +\left\{\epsilon_{\mu}^{*}\left(m_{B}^{2}-m_{V}^{2}\right)-\left(\epsilon^{*} q\right)(2 p+q)_{\mu}\right\} T_{2}^{B V}\left(q^{2}\right) \\
& +\left(\epsilon^{*} q\right)\left\{q_{\mu}-\frac{q^{2}}{m_{B}^{2}-m_{V}^{2}}(2 p+q)_{\mu}\right\} T_{3}^{B V}\left(q^{2}\right) .
\end{aligned}
$$

In the above, $\kappa=\sqrt{2}(\kappa=1)$ for $\pi^{0}$ and $\rho^{0}$ (for other mesons).

In what follows, we derive new LCSR for the $B \rightarrow P, V$ form factors listed in Table 1. For definiteness, we assume the following flavour configurations: $\bar{B}_{d}^{0} \rightarrow \pi^{+}, \rho^{+}$and $\bar{B}_{d}^{0} \rightarrow \bar{K}^{0}, \bar{K}^{* 0}$. The sum rules for the remaining form factors $f_{B P}^{0}, A_{0}^{B V}$ and $T_{2,3}^{B V}$, will be presented elsewhere. Importantly, in all channels considered in this paper, the threshold parameters $s_{0}^{P, V}$ can be obtained from the two-point sum rules for the decay constants $f_{P, V}$.

Let us now calculate the r.h.s. of the sum rule (12). As explained in the previous section, the leading-order contribution to the OPE is given by the diagram in Fig. 1a. The answer is obtained, by decomposing the matrix element in Eq. (8) at $x^{2}=0$ :

$$
\begin{aligned}
& \left\langle 0\left|\bar{q}_{2 \alpha}(x)[x, 0] h_{v \beta}(0)\right| \bar{B}_{v}\right\rangle \\
& =-\frac{i f_{B} m_{B}}{4} \int_{0}^{\infty} d \omega e^{-i \omega v \cdot x}\left[(1+\psi)\left\{\phi_{+}^{B}(\omega)-\frac{\phi_{+}^{B}(\omega)-\phi_{-}^{B}(\omega)}{2 v \cdot x} \not x\right\} \gamma_{5}\right]_{\beta \alpha}
\end{aligned}
$$

in terms of the $B$-meson two-particle DA's $\phi_{+}^{B}(\omega)$ and $\phi_{-}^{B}(\omega)$ defined [9, 12] in the momentum space. In the above, $[x, 0]$ is the path-ordered gauge factor. The variable $\omega>0$ is the plus component of the spectator-quark momentum in the $B$ meson. Substituting Eq. (17) in Eq. (8) and integrating over $x$, one obtains the invariant amplitudes $F^{(B), O P E}\left(p^{2}, q^{2}\right)$ which have a simple generic expression at $q^{2}=0$ :

$$
F^{(B), O P E}\left(p^{2}, 0\right)=\sum_{n=1,2} \int_{0}^{\infty} \frac{d \omega \phi_{n}(\omega)}{\left[\left(1-\omega / m_{B}\right)\left(\omega m_{B}-p^{2}\right)\right]^{n}},
$$


where the functions $\phi_{n}(\omega)$ are combined from the $B$-meson DA's. If one continues the $x^{2}$-expansion of the matrix element (17) beyond the leading order, the resulting contributions to $F^{(B), O P E}$ will be suppressed by additional powers of the denominator (i.e., by inverse powers of $M^{2}$ after Borel transformation). We neglect them, having assumed that $P^{2}=-p^{2}$ (or $M^{2}$ ) is a large scale. Furthermore, $B$-meson DA's are essentially concentrated around $\omega \sim \bar{\Lambda}$, where $\bar{\Lambda}=m_{B}-m_{b}$, with the kinematical limit $\omega<2 \bar{\Lambda}$. Hence, the denominator in Eq. (18) implicitly contains another large scale $m_{B} \bar{\Lambda}$. The heavy-mass scale which reappears in the HQET correlation function, has a kinematical origin: at $q^{2}=0$ the external momenta $p$ and $q$ are both $O\left(m_{b} / 2\right)$, or in other words, as already mentioned in the previous section, the rescaled virtuality $\tilde{q}^{2}=O\left(m_{b} \bar{\Lambda}\right)$. Finally, to obtain the r.h.s. of Eq. (12), one transforms the integral in Eq. (18) into a dispersion form, changing the variable $\omega$ to $s=\omega m_{B}$, performing the Borel transformation in the variable $p^{2}$ and replacing the upper limit by the duality threshold $s_{0}^{P, V}$. Importantly, due to the fact that $\sqrt{s_{0}^{P, V}} \ll m_{B}$ only the regions of small momenta of spectator quark $\omega<s_{0}^{P, V} / m_{B}$, far from the kinematical threshold $\omega \sim \bar{\Lambda}$ are important in the LCSR. As already mentioned in [7, this situation corresponds to the end-point mechanism which is realized in heavy-to-light exclusive transitions in the absence of hard-gluon exchanges.

Following the derivation described above, we obtain the leading-order LCSR for the $B \rightarrow \pi, \rho$ form factors at zero momentum transfer $\left(q^{2}=0\right)$, where the $u, d$-quark masses are neglected, and the pion mass is put to zero:

$$
\begin{gathered}
f_{B \pi}^{+}(0)=\frac{f_{B}}{f_{\pi} m_{B}} \int_{0}^{s_{0}^{\pi}} d s e^{-s / M^{2}} \phi_{-}^{B}\left(s / m_{B}\right) \\
f_{B \pi}^{+}(0)+f_{B \pi}^{-}(0)=\frac{f_{B}}{f_{\pi} m_{B}} \int_{0}^{s_{0}^{\pi}} d s e^{-s / M^{2}}\left[\frac{m_{B}^{2}}{m_{B}^{2}-s} \phi_{+}^{B}\left(s / m_{B}\right)\right. \\
\left.-2 \frac{s}{m_{B}^{2}-s} \phi_{-}^{B}\left(s / m_{B}\right)+2 \frac{m_{B}^{3}}{\left(m_{B}^{2}-s\right)^{2}} \bar{\Phi}_{ \pm}^{B}\left(s / m_{B}\right)\right] \\
f_{B \pi}^{T}(0)=\frac{f_{B}}{f_{\pi} m_{B}} \int_{0}^{s_{0}^{\pi}} d s e^{-s / M^{2}}\left[\phi_{-}^{B}\left(s / m_{B}\right)\right. \\
\left.-\phi_{+}^{B}\left(s / m_{B}\right)-\frac{m_{B}}{m_{B}^{2}-s} \bar{\Phi}_{ \pm}^{B}\left(s / m_{B}\right)\right] \\
V^{B \rho}(0)=\frac{f_{B}\left(m_{B}+m_{\rho}\right)}{2 f_{\rho} m_{\rho} m_{B}} e^{m_{\rho}^{2} / M^{2}} \int_{0}^{s_{0}^{\rho}} d s e^{-s / M^{2}} \frac{m_{B}^{2}}{m_{B}^{2}-s} \phi_{+}^{B}\left(s / m_{B}\right) \\
A_{1}^{B \rho}(0)=\frac{f_{B} m_{B}}{2 f_{\rho} m_{\rho}\left(m_{B}+m_{\rho}\right)} e^{m_{\rho}^{2} / M^{2}} \int_{0}^{s_{0}^{\rho}} d s e^{-s / M^{2}} \phi_{+}^{B}\left(s / m_{B}\right)
\end{gathered}
$$




$$
\begin{gathered}
A_{2}^{B \rho}(0)=\frac{f_{B}}{2 f_{\rho} m_{\rho}} \frac{\left(m_{B}+m_{\rho}\right)}{m_{B}} e^{m_{\rho}^{2} / M^{2}} \int_{0}^{s_{0}^{\rho}} d s e^{-s / M^{2}} \\
{\left[\frac{m_{B}^{2}}{m_{B}^{2}-s} \phi_{+}^{B}\left(s / m_{B}\right)-2 \frac{s}{m_{B}^{2}-s} \phi_{-}^{B}\left(s / m_{B}\right)+2 \frac{m_{B}^{3}}{\left(m_{B}^{2}-s\right)^{2}} \bar{\Phi}_{ \pm}^{B}\left(s / m_{B}\right)\right]} \\
T_{1}^{B \rho}(0)=\frac{f_{B}}{2 f_{\rho} m_{\rho}} e^{m_{\rho}^{2} / M^{2}} \int_{0}^{s_{0}^{\rho}} d s e^{-s / M^{2}} \phi_{+}^{B}\left(s / m_{B}\right)
\end{gathered}
$$

where a compact notation:

$$
\bar{\Phi}_{ \pm}^{B}(\omega)=\int_{0}^{\omega} d \tau\left(\phi_{+}^{B}(\tau)-\phi_{-}^{B}(\tau)\right)
$$

is introduced. The Borel parameter $M$ in the light-meson channels has typical values around $1 \mathrm{GeV}$, still $M \gg \Lambda_{Q C D}$. The first sum rule (19) has already been derived in [7] (see also [8]), whereas all other sum rules are new. The LCSR at $q^{2} \neq 0$ and $m_{q_{1}} \neq 0$ have bulky expressions presented in the Appendix. Substituting $m_{q_{1}}=m_{s}$ and replacing $s_{0}^{\pi, \rho} \rightarrow s_{0}^{K, K^{*}}$, one obtains LCSR for the $B \rightarrow K, K^{*}$ form factors.

In this paper we neglect $O\left(\alpha_{s}\right)$ radiative corrections due to the hard-gluon exchanges between the quark-antiquark lines (one of the diagrams is shown in Fig. 1c). Their calculation is inseparable from the nontrivial renormalization of $B$-meson DA's, which is so far known only for $\phi_{+}^{B}(\omega)$ [19] (for a detailed discussion see also [17, 11]). As far as the normalization scale of $\phi_{+}^{B}(\omega)$ or its inverse moment

$$
\frac{1}{\lambda_{B}(\mu)}=\int_{0}^{\infty} d \omega \frac{\phi_{+}^{B}(\omega, \mu)}{\omega}
$$

is concerned, we assume that $\mu \simeq M$, having in mind that the Borel scale reflects the average virtuality in the correlator.

In addition we calculate the corrections due to the three-particle (quarkantiquark-gluon) DA's of the $B$ meson. They correspond to the diagram in Fig. 1b, where a low virtuality gluon is emitted from the virtual quark and absorbed in the $B$ meson. The contribution of this diagram to the correlation function (1) is obtained by contracting the $q_{1}(x)$ and $\bar{q}_{1}(0)$ fields and inserting the one-gluon part of the quark propagator near the light-cone [22]:

$$
S_{q_{1}}\left(x, 0, m_{q_{1}}\right)=\int \frac{d^{4} p}{(2 \pi)^{4}} e^{-i p x} \int_{0}^{1} d v G_{\mu \nu}(v x)\left[\frac{v x^{\mu} \gamma^{\nu}}{p^{2}-m_{q_{1}}^{2}}-\frac{\left(\not p+m_{q_{1}}\right) \sigma^{\mu \nu}}{2\left(p^{2}-m_{q_{1}}^{2}\right)^{2}}\right],
$$

where $G_{\mu \nu}=g_{s} G_{\mu \nu}^{a}\left(\lambda^{a} / 2\right)$. As a result, an expression similar to Eq. (8) emerges with the vacuum-to- $B$ matrix element containing a nonlocal product of quark, antiquark and gluon fields. In $x^{2}=0$ limit we adopt the following decomposition 
of this matrix element into four independent three-particle DA's:

$$
\begin{aligned}
& \left\langle 0\left|\overline{q_{2}}(x) G_{\lambda \rho}(u x) h_{v \beta}(0)\right| \bar{B}^{0}(v)\right\rangle=\frac{f_{B} m_{B}}{4} \int_{0}^{\infty} d \omega \int_{0}^{\infty} d \xi e^{-i(\omega+u \xi) v \cdot x} \\
& \times\left[( 1 + \psi ) \left\{\left(v_{\lambda} \gamma_{\rho}-v_{\rho} \gamma_{\lambda}\right)\left(\Psi_{A}(\omega, \xi)-\Psi_{V}(\omega, \xi)\right)-i \sigma_{\lambda \rho} \Psi_{V}(\omega, \xi)\right.\right. \\
& \left.\left.-\left(\frac{x_{\lambda} v_{\rho}-x_{\rho} v_{\lambda}}{v \cdot x}\right) X_{A}(\omega, \xi)+\left(\frac{x_{\lambda} \gamma_{\rho}-x_{\rho} \gamma_{\lambda}}{v \cdot x}\right) Y_{A}(\omega, \xi)\right\} \gamma_{5}\right]_{\beta \alpha},
\end{aligned}
$$

where the path-ordered gauge factors on l.h.s. are omitted for brevity. Multiplying both parts of this expression by $x^{\rho}$ one encounters the definition introduced in [16]. The DA's $\Psi_{V}, \Psi_{A}, X_{A}$ and $Y_{A}$ depend on the two variables $\omega>0$ and $\xi>0$ being, respectively, the plus components of the light-quark and gluon momenta in the $B$ meson.

Our analysis in this paper is restricted to the four three-particle DA's defined in Eq. (28), and to the two-particle DA's defined in Eq. (17). One can further expand both matrix elements near the light-cone in powers of $x^{2}$ introducing additional DA's 1. As argued above, their contributions to the correlation function will be power-suppressed, at least by inverse powers of $M^{2}$.

The resulting expressions for the three-particle contributions to LCSR at $q^{2} \neq 0$ are presented in the Appendix. At $q^{2}=0$ these expressions (which we do not display for brevity) have to be added to the leading-order sum rules (19) -(25). The new LCSR are sensitive to the normalization constants and to the behavior of the B-meson two-particle (three-particle) DA's at small $\omega(\omega, \xi)$, hence, also to the inverse moment $\lambda_{B}$. For the two-particle DA's the behavior at $\omega \rightarrow 0$ is known, and we have at our disposal models for $\phi_{ \pm}^{B}(\omega)$ [9, 17, 18. The remaining task is to establish the behavior of the three-particle DA's at small $\omega, \xi$, and to build a model for them.

\section{Three-particle DA's from sum rules in HQET}

As already mentioned, QCD sum rules in HQET were employed in [9, 17] to predict the $B$-meson two-particle DA's $\phi_{ \pm}^{B}(\omega)$. The idea was to introduce a correlation function with two $\bar{q} \Gamma h_{v}$ currents, one of them local and the other one containing the $h_{v}$ and $\bar{q}$ fields at a light-like separation. The ground $B_{v}$-state contribution to the hadronic dispersion relation for this correlation function contains the product of the $B$-meson decay constant and the nonlocal heavy-to-light matrix element (17). An appropriate choice of the Dirac-structure $\Gamma$ allows one to separate $\phi_{+}^{B}$ from $\phi_{-}^{B}$. Matching the $B$-meson term to the leading perturbative contribution (the loop diagram) via quark-hadron duality, one reproduces [9] the behavior of both DA's at $\omega \rightarrow 0: \phi_{+}^{B}(\omega) \sim \omega$ and $\phi_{-}^{B}(\omega) \sim$ const, in accordance with general expectations.

Here we use a similar method and derive HQET sum rules for the $B$ meson three-particle DA's in the perturbative loop approximation. The starting object

\footnotetext{
${ }^{1}$ Recently, a more general decomposition of the three-particle matrix element (28) was suggested in 23, where one encounters additional three-particle DA's. A separate study is needed to clarify the importance of these amplitudes with respect to the main four threeparticle DA's.
} 


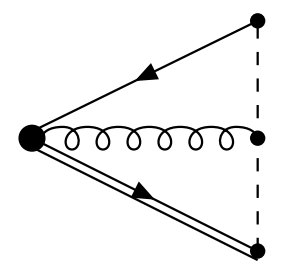

Figure 2: Perturbative loop diagram for the correlation function (29). The points connected with the dashed line (the thick point) represent the vertex of the nonlocal (local) current.

is the correlation function

$$
\begin{aligned}
\Pi_{\lambda}^{(\Gamma)}(\rho, t, u)= & i \int d^{4} y e^{-i \rho(v \cdot y)} \\
& \left\langle 0\left|T\left\{\bar{q}(t n) \Gamma G_{\lambda \sigma}(u t n) n^{\sigma} h_{v}(0), \bar{h}_{v}(y) G_{\alpha \beta} \sigma^{\alpha \beta} \gamma_{5} q(y)\right\}\right| 0\right\rangle,
\end{aligned}
$$

where the local current containing the effective heavy-quark, light-antiquark and gluon fields is correlated with a generic nonlocal current, with all three fields on the light-cone. We define the light-like unit vectors $n_{\mu}$ and $\bar{n}_{\mu}\left(n^{2}=\bar{n}^{2}=\right.$ $0, n \cdot \bar{n}=2)$ so that $v_{\mu}=\left(n_{\mu}+\bar{n}_{\mu}\right) / 2 ; t$ is an arbitrary real number, determining the location on the light-cone (that is, $t n$ corresponds to the light-like interval $x$ in Eq. (28)) and $\rho$ is the "off-shell energy", the HQET analog of virtuality. The gauge factors between the fields in Eq. (29) are omitted for brevity, in fact they are inessential for the perturbative loop approximation. The local current in (29) is chosen in a convenient scalar form, note that other choices are also possible.

The correlation function (29), after inserting the complete set of hadronic states, has a pole of the $B_{v}$ state at $\rho=\bar{\Lambda}$ where $\bar{\Lambda}=m_{B}-m_{b}$, schematically:

$$
\Pi_{\lambda}^{(\Gamma)}(\rho, t, u)=\frac{C_{\lambda}}{\bar{\Lambda}-\rho} \int_{0}^{\infty} d \omega \int_{0}^{\infty} d \xi e^{-i(\omega+u \xi) t} F(\omega, \xi),
$$

where $C_{\lambda}$ is proportional to the hadronic matrix element of the local current in Eq. (29) and to other normalization constants; since we are only interested in the functional dependence on $\omega$ and $\xi$, this factor does not need to be specified. $F(\omega, \xi)$ is one of the three-particle DA's $\Psi_{A}(\omega, \xi), \Psi_{V}(\omega, \xi), X_{A}(\omega, \xi), Y_{A}(\omega, \xi)$ (or their linear combination), depending on the choice of the Dirac-structure in the nonlocal current in Eq. (29). More specifically, the following correspondence is established:

$$
\begin{array}{rlr}
\Gamma=\gamma_{\mu} \gamma_{5} & \rightarrow F=\Psi_{A} \\
\gamma_{\mu} & \rightarrow & \Psi_{V} \\
\sigma_{\mu \nu} \gamma_{5} & \rightarrow & \Psi_{A}-\Psi_{V} \\
\not h \gamma_{5} & \rightarrow & X_{A}+\Psi_{A} \\
i \gamma_{5} & \rightarrow & Y_{A}-X_{A} .
\end{array}
$$

To proceed, we calculate the spectral density of the leading-order perturbative contribution to the correlation function (29), given by the loop diagram 
in Fig. 2. All three intermediate lines in this diagram have to be put on-shell, which simplifies the calculation. Substituting free propagators for the effective heavy-quark, light-quark and gluon fields, we use Cutkosky rules and obtain a dispersion relation for the Fig. 2 diagram contribution to the correlation function

$$
\begin{gathered}
\Pi_{\lambda}^{(\Gamma), p e r t}(\rho, t, u)=\tilde{c} \int_{0}^{\infty} \frac{d s}{s-\rho} \int \frac{d^{4} l d^{4} k}{(2 \pi)^{8}} e^{-i t(l+u k) \cdot n}\left(k_{\lambda} k_{\alpha} n_{\beta}+g_{\lambda \alpha} k_{\beta} n \cdot k\right) \\
\delta(s-(l \cdot v+k \cdot v)) \delta\left(l^{2}\right) \delta\left(k^{2}\right) \Theta\left(l_{0}\right) \Theta\left(k_{0}\right) \operatorname{Tr}\left[\Gamma \frac{1+\psi}{2} \sigma^{\alpha \beta} \gamma_{5} l\right], \quad \text { (32) }
\end{gathered}
$$

where $l$ and $k$ are the four-momenta of the light-quark and gluon lines, respectively, and $\tilde{c}$ is the constant factor (containing also $\alpha_{s}$ ). The integration is conveniently carried out if one expands these momenta in light-cone components using the basis of the light-like vectors $n$ and $\bar{n}$ introduced above:

$$
\begin{aligned}
k_{\mu} & =\frac{1}{2}\left[(k \cdot \bar{n}) n_{\mu}+(k \cdot n) \bar{n}_{\mu}\right]+k_{\perp \mu}, \\
l_{\mu} & =\frac{1}{2}\left[(l \cdot \bar{n}) n_{\mu}+(l \cdot n) \bar{n}_{\mu}\right]+l_{\perp \mu} .
\end{aligned}
$$

The delta-functions in Eq. (32) are integrated out, taking into account the kinematical bounds represented by $\Theta$-functions. At the end two integrations are left, with the variables $\omega=(l \cdot n)$ and $\xi=(k \cdot n)$, that is, the plus components of the quark and gluon loop momenta, respectively. Matching the result of this calculation to the hadronic dispersion relation with the pole-term (30) and employing quark-hadron duality for the excited and continuum states with an effective threshold $\tilde{s}_{0}$, we perform the Borel transformation. Comparing the dependence on the variables $\omega$ and $\xi$ on both sides, the following sum rules for three-particles DA's are obtained, in the perturbative loop approximation:

$$
\begin{aligned}
& \Psi_{A}(\omega, \xi)=\Psi_{V}(\omega, \xi)=r \xi^{2} \int_{\substack{(\xi+\omega) / 2 \\
\tilde{s}_{0}}}^{\tilde{s}^{2}} d s e^{(-s+\bar{\Lambda}) / \tau} \\
& X_{A}(\omega, \xi)=r \xi(2 \omega-\xi) \int_{(\xi+\omega) / 2}^{\tilde{s}_{0}} \times(2 s-\omega)^{2} \Theta\left(2 \tilde{s}_{0}-\omega-\xi\right)+\ldots \\
& Y_{A}(\omega, \xi)=r \xi \int_{(\xi+\omega) / 2}^{(-s+\bar{\Lambda}) / \tau}(2)^{2}\left(2 \tilde{s}_{0}-\omega-\xi\right)+\ldots \\
& \times(2 s-\omega-\xi)^{2}\left(-\frac{(2 s-\omega-\xi)}{3}+3 \omega-\xi\right) \Theta\left(2 \tilde{s}_{0}-\omega-\xi\right)+\ldots
\end{aligned}
$$


with an equal coefficient $r$ emerging from the constant factors $C_{\lambda}$ and $\tilde{c}$ in Eqs. (30) and (32), respectively. Importantly, in the loop approximation, $\Psi_{V}(\omega, \xi)$ and $\Psi_{A}(\omega, \xi)$ are equal, while $X_{A}$ and $Y_{A}$ have different forms. If one takes the local limit $t \rightarrow 0$ of the correlation function (29), the resulting two-point sum rules for the normalization constants of the DA's yield, $\int_{0}^{\infty} d \omega d \xi \Psi_{A}(\omega, \xi)=$ $\int_{0}^{\infty} d \omega d \xi \Psi_{V}(\omega, \xi)$.

In fact, $\Psi_{A}(\omega, \xi)$ and $\Psi_{V}(\omega, \xi)$ have independent normalization conditions

$$
\begin{aligned}
& \int_{0}^{\infty} d \omega \int_{0}^{\infty} d \xi \Psi_{A}(\omega, \xi)=\frac{\lambda_{E}^{2}}{3} \\
& \int_{0}^{\infty} d \omega \int_{0}^{\infty} d \xi \Psi_{V}(\omega, \xi)=\frac{\lambda_{H}^{2}}{3}
\end{aligned}
$$

where the constants $\lambda_{E}$ and $\lambda_{H}$ are determined by the matrix elements of different local operators [9]. The functions $X$ and $Y$ are normalized to zero, but can also have different normalization coefficients.

The differences for all four DA's manifest themselves if in the sum rules (34)-(36) one takes into account the condensate contributions (indicated by ellipses), suppressed by the inverse powers of the Borel scale $\tau$. In fact, in the correlation functions with nonlocal currents the usual approximation of local quark and gluon condensates is too crude and models of nonlocal condensates are usually employed $[9,17$. We have investigated only the local limit of the correlation function (29) and the resulting sum rules for $\lambda_{E}$ and $\lambda_{H}$. The condensate contributions have indeed different sizes in these sum rules, but their influence on the normalization constants is moderate, as compared with the loop contribution. The details of this analysis will be presented elsewhere. Note that the sum rules for $\lambda_{E}, \lambda_{H}$ derived in 9 are based on a different, "nondiagonal" correlator with one three-particle and one two-particle current. These sum rules predict

$$
\lambda_{E}^{2}=(0.11 \pm 0.06) \mathrm{GeV}^{2}, \quad \lambda_{H}^{2}=(0.18 \pm 0.07) \mathrm{GeV}^{2},
$$

not very far from each other, indicating that the approximation $\lambda_{E}=\lambda_{H}$ which follows from (34) can be adopted within the uncertainty intervals in Eq. (38).

The most important prediction of the sum rules (34)-(36), is the behavior at $\omega, \xi \rightarrow 0$ given by the perturbative loop contribution:

$$
\begin{aligned}
\Psi_{A}(\omega, \xi) \sim \Psi_{V}(\omega, \xi) & \sim \xi^{2}, \\
X_{A}(\omega, \xi) & \sim \xi(2 \omega-\xi), \quad Y_{A} \sim \xi .
\end{aligned}
$$

In our previous paper [7, we followed a different, more qualitative way, making a comparison between the pion and $B$-meson three-particle DA's in the asymptotic regime and obtained

$$
\left(\Psi_{A}-\Psi_{V}\right) \sim\left(\lambda_{E}^{2}-\lambda_{H}^{2}\right) \omega \xi^{2},
$$

which turns out to be a small correction, neglected here. This correction does not contradict the behavior indicated in Eq. (39) but cannot be simply extracted from the sum rules (34) without going beyond the loop approximation. 
We suggest two models for the three-particle DA's. The first one is obtained from the sum rules (34)-(36) in the local duality (LD) $\tau \rightarrow \infty$ limit:

$$
\begin{aligned}
\Psi_{A}^{L D}(\omega, \xi) & =\Psi_{V}^{L D}(\omega, \xi)=\left(\frac{35 \lambda_{E}^{2}}{4 \tilde{s}_{0}^{4}}\right) \xi^{2}\left(1-\frac{\omega+\xi}{2 \tilde{s}_{0}}\right)^{3} \Theta\left(2 \tilde{s}_{0}-\omega-\xi\right), \\
X_{A}^{L D}(\omega, \xi) & =\left(\frac{35 \lambda_{E}^{2}}{4 \tilde{s}_{0}^{4}}\right) \xi(2 \omega-\xi)\left(1-\frac{\omega+\xi}{2 \tilde{s}_{0}}\right)^{3} \Theta\left(2 \tilde{s}_{0}-\omega-\xi\right), \\
Y_{A}^{L D}(\omega, \xi) & =-\left(\frac{35 \lambda_{E}^{2}}{16 \tilde{s}_{0}^{4}}\right) \xi\left(1-\frac{\omega+\xi}{2 \tilde{s}_{0}}\right)^{3}\left(2 \tilde{s}_{0}-13 \omega+3 \xi\right) \Theta\left(2 \tilde{s}_{0}-\omega-\xi\right) .
\end{aligned}
$$

The uniform constant factor in the above expressions is fixed by the normalization conditions (37), and we assume that $\lambda_{E}=\lambda_{H}$. Note that $X_{A}(\omega, \xi)$ and $Y_{A}(\omega, \xi)$ in Eqs. (35), (36) are normalized to zero, as they should be.

It is natural to combine the above three-particle DA's with $\phi_{ \pm}^{B}(\omega)$ obtained in the same local-duality limit from the HQET sum rule for a correlator of the nonlocal and local quark-antiquark currents:

$$
\begin{aligned}
\phi_{+}^{B, L D}(\omega) & =\frac{3 \omega}{2 \tilde{s}_{0}^{2}}\left(1-\frac{\omega}{2 \tilde{s}_{0}}\right) \Theta\left(2 \tilde{s}_{0}-\omega\right), \\
\phi_{-}^{B, L D}(\omega) & =\frac{3}{2 \tilde{s}_{0}}\left(1-\frac{\omega}{2 \tilde{s}_{0}}\right)^{2} \Theta\left(2 \tilde{s}_{0}-\omega\right),
\end{aligned}
$$

where $\phi_{+}^{B, L D}$ has already been derived in [17].

For the second model of the three-particle DA's we combine the small $\omega, \xi$ behavior (39) with an exponential fall-off:

$$
\begin{aligned}
\Psi_{A}(\omega, \xi) & =\Psi_{V}(\omega, \xi)=\frac{\lambda_{E}^{2}}{6 \omega_{0}^{4}} \xi^{2} e^{-(\omega+\xi) / \omega_{0}}, \\
X_{A}(\omega, \xi) & =\frac{\lambda_{E}^{2}}{6 \omega_{0}^{4}} \xi(2 \omega-\xi) e^{-(\omega+\xi) / \omega_{0}} \\
Y_{A}(\omega, \xi) & =-\frac{\lambda_{E}^{2}}{24 \omega_{0}^{4}} \xi\left(7 \omega_{0}-13 \omega+3 \xi\right) e^{-(\omega+\xi) / \omega_{0}} .
\end{aligned}
$$

The analogous ansatz for the two-particle DA's was suggested in 9 :

$$
\begin{aligned}
\phi_{+}^{B}(\omega) & =\frac{\omega}{\omega_{0}^{2}} e^{-\frac{\omega}{\omega_{0}}}, \\
\phi_{-}^{B}(\omega) & =\frac{1}{\omega_{0}} e^{-\frac{\omega}{\omega_{0}}},
\end{aligned}
$$

so that $\lambda_{B}=\omega_{0}$.

After the models are formulated, it is important to check if they obey the constraints derived in [16] (see also [12]) from the QCD equations of motion (adapted to HQET) in the form of two equations for the two-particle DA's:

$$
\begin{aligned}
\omega \frac{d \phi_{-}^{B}(\omega)}{d \omega}+\phi_{+}^{B}(\omega) & =I(\omega), \\
(\omega-2 \bar{\Lambda}) \phi_{+}^{B}(\omega)+\omega \phi_{-}^{B}(\omega) & =J(\omega),
\end{aligned}
$$


where $I(\omega)$ and $J(\omega)$ are the 'source' terms due to the three-particle DA's:

$$
\begin{aligned}
I(\omega)= & 2 \frac{d}{d \omega} \int_{0}^{\omega} d \rho \int_{\omega-\rho}^{\infty} \frac{d \xi}{\xi} \frac{\partial}{\partial \xi}\left[\Psi_{A}(\rho, \xi)-\Psi_{V}(\rho, \xi)\right], \\
J(\omega)= & -2 \frac{d}{d \omega} \int_{0}^{\omega} d \rho \int_{\omega-\rho}^{\infty} \frac{d \xi}{\xi}\left[\Psi_{A}(\rho, \xi)+X_{A}(\rho, \xi)\right] \\
& -4 \int_{0}^{\omega} d \rho \int_{\omega-\rho}^{\infty} \frac{d \xi}{\xi} \frac{\partial \Psi_{V}(\rho, \xi)}{\partial \xi} .
\end{aligned}
$$

We immediately notice that $I(\omega)=0$ in both models (41) and (43). In other words, the relation 12 .

$$
\phi_{-}^{B}(\omega)=\int_{\omega}^{\infty} d \omega \frac{\phi_{+}^{B}(\omega)}{\omega}
$$

does not receive gluon corrections in the approximation adopted here. Importantly, within this approximation also $J(0)=0$, hence the behavior $\phi_{+}^{B}(\omega) \sim \omega$ at $\omega \rightarrow 0$ is not modified, contrary to a general expectation [16.

In addition, due to the relations between the matrix elements of local operators the moments of DA's have to fulfil [9] the following equations: 2

$$
\begin{aligned}
\langle\omega\rangle_{+} & =\frac{4}{3} \bar{\Lambda}, \quad\langle\omega\rangle_{-}=\frac{2}{3} \bar{\Lambda}, \\
\left\langle\omega^{2}\right\rangle_{+} & =2 \bar{\Lambda}^{2}+\frac{2}{3} \lambda_{E}^{2}+\frac{1}{3} \lambda_{H}^{2}, \\
\left\langle\omega^{2}\right\rangle_{-} & =\frac{2}{3} \bar{\Lambda}^{2}+\frac{1}{3} \lambda_{H}^{2},
\end{aligned}
$$

where $\left\langle\omega^{n}\right\rangle_{ \pm} \equiv \int_{0}^{\infty} d \omega \phi_{ \pm}(\omega) \omega^{n}$.

For the DA's of the local-duality model (42) and (41), only the first equation in (45) is valid. Hence, the 2-particle DA's acquire corrections determined by the three-particle DA's:

$$
\phi_{ \pm}^{B, L D}(\omega) \rightarrow \phi_{ \pm}^{B, L D}(\omega)+\delta \phi_{ \pm}^{B, L D}(\omega) .
$$

Instead of substituting Eq. (49) into Eqs. (45) and solving the system of two equations for the functions $\delta \phi_{ \pm}^{B, L D}(\omega)$, it is easier to use the ansatz obtained in [16]:

$$
\begin{aligned}
\phi_{+}^{B, L D}(\omega)+\delta \phi_{+}^{B, L D}(\omega) & =\phi_{+}^{B, W W}(\omega)+\frac{\omega}{2 \bar{\Lambda}} \Phi(\omega), \\
\phi_{-}^{B, L D}(\omega)+\delta \phi_{-}^{B, L D}(\omega) & =\phi_{-}^{B, W W}(\omega)+\frac{2 \bar{\Lambda}-\omega}{2 \bar{\Lambda}} \Phi(\omega)+\frac{J(\omega)}{\omega},
\end{aligned}
$$

where $\phi_{+}^{B, W W}(\omega)$ are the solutions of Eqs. (45) without the 'source' terms $I(\omega)$ and $J(\omega)$ (in Wandzura-Wilsczek (WW) approximation):

$$
\begin{aligned}
\phi_{+}^{B, W W}(\omega) & =\frac{\omega}{2 \bar{\Lambda}^{2}} \Theta(2 \bar{\Lambda}-\omega), \\
\phi_{-}^{B, W W}(\omega) & =\frac{2 \bar{\Lambda}-\omega}{2 \bar{\Lambda}^{2}} \Theta(2 \bar{\Lambda}-\omega),
\end{aligned}
$$

\footnotetext{
${ }^{2}$ Note that after including renormalization effects in DA's, which so far have only been studied for $\phi_{+}^{B}[19$, the positive moments of DA's logarithmically diverge, and have to be regularized in some way.
} 
and $\Phi(\omega)$ has a complicated expression via $I(\omega)$ and $J(\omega)$ which can be found in 16. We have calculated $J(\omega)$ for the local-duality model (41) and the resulting function $\Phi(\omega)$. The results for $\phi_{ \pm}^{B, L D}(\omega)+\delta \phi_{ \pm}^{B, L D}(\omega)$ obtained from Eqs. (50) are shown in Fig. 3. The corrected DA's differ significantly from the WWapproximation, in particular, $\left(1 / \lambda_{B}\right)^{W W}=1 / \bar{\Lambda}$ is shifted to $1 / \lambda_{B}=1 / \bar{\Lambda}+$ $7 \lambda_{E}^{2} /\left(2 \bar{\Lambda} \tilde{s}_{0}^{2}\right)$, while the positive moments satisfy Eqs. (48)) (by construction of the ansatz (50)). After including the gluon corrections, the functions $\phi_{ \pm}^{B}(\omega)$ become smoother and are shifted towards lower $\omega$ 's, as expected..

Turning to the exponential model and substituting Eqs. (43) for $\Psi_{A}$ and $X_{A}$ into Eq. (46), we obtain:

$$
J(\omega)=\frac{2 \lambda_{E}^{2}}{3 \omega_{0}^{4}} \omega\left(\omega-2 \omega_{0}\right) e^{-\frac{\omega}{\omega_{0}}} .
$$

In this case, if the conditions [9]:

$$
\omega_{0}=\frac{2}{3} \bar{\Lambda}, \quad \lambda_{E}^{2}=\lambda_{H}^{2}=\frac{3}{2} \omega_{0}^{2}=\frac{2}{3} \bar{\Lambda}^{2} .
$$

are satisfied, both equations in (45) can be solved, and the solution for the two-particle DA's has the exponential form (44). Under the same conditions, the relations (48) between the moments are also fulfilled, as already noticed in [9. Hence, the three-particle DA's described by the exponential model (43) do not induce additional corrections to the ansatz (44). We conclude that the combination of Eqs. (44) and (43), together with the conditions (53) form a selfconsistent model of two- and three-particle $B$ meson DA's.

Comparing the exponential model with the local-duality one introduced above, we find that numerically, in the region of integration in LCSR , $\omega<$ $s_{0}^{\pi\left(K, \rho, K^{*}\right)} / m_{B}$, the two models for $\phi_{ \pm}^{B}$ are almost indistinguishable (if $\lambda_{E}=\lambda_{H}$ and $\lambda_{B}$ are the same), as can be seen from Fig. 3. For that reason, in the numerical analysis of LCSR, we only consider the exponential model.
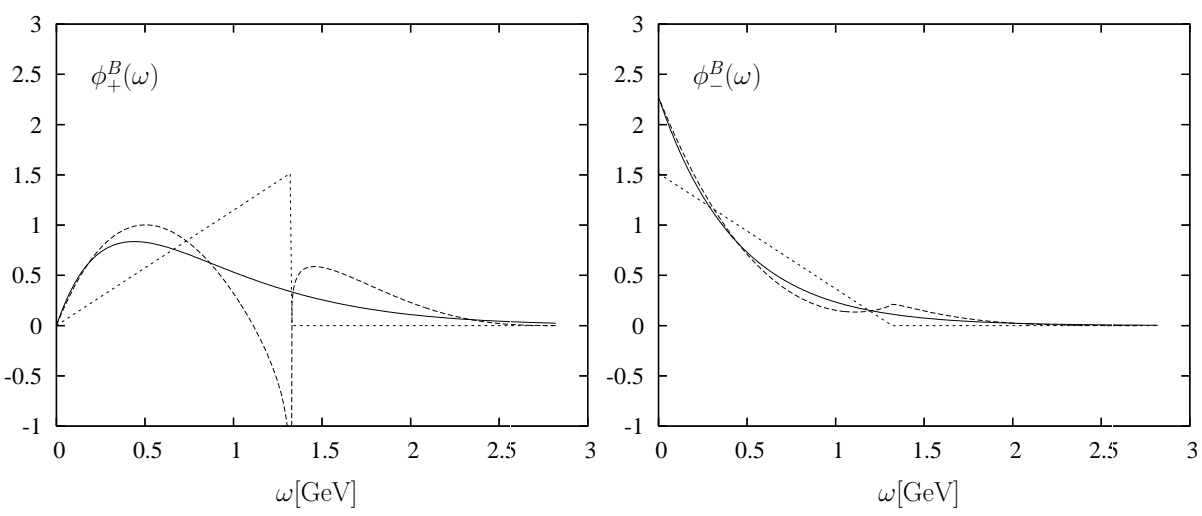

Figure 3: B-meson two-particle DA's $\phi_{+}^{B}(\omega)$ (left) and $\phi_{-}^{B}(\omega)$ (right). The solid (dashed) lines represent the exponential (local-duality) model; short-dashed lines are the $W W$-parts (51). In both models $\lambda_{B}=\frac{2}{3} \bar{\Lambda}$ and $\lambda_{E}^{2}=\lambda_{H}^{2}=\frac{2}{3} \bar{\Lambda}^{2}$.

In the region of small $\omega$ the exponential ansatz (44) for $\phi_{+}^{B}(\omega)$ is numerically 
close to the more elaborated model suggested in [17]:

$$
\phi_{+}^{B}(\omega, \mu=1 \mathrm{GeV})=\frac{4 \omega}{\pi \lambda_{B}\left(1+\omega^{2}\right)}\left[\frac{1}{1+\omega^{2}}-\frac{2\left(\sigma_{B}-1\right)}{\pi^{2}} \ln (\omega)\right],
$$

provided $\omega_{0}=\lambda_{B}$. In the above, $\omega$ is in $\mathrm{GeV}$ units and the parameters $\lambda_{B}$ and $\sigma_{B}$ are determined from HQET sum rules including the QCD radiative and nonperturbative corrections. Also the model for $\phi_{+}^{B}(\omega)$ suggested in [18] at small $\omega$ contains the same exponential component as in Eq. (44).

\section{$5 \quad$ Heavy-mass limit of LCSR}

In this section we discuss the power counting in the $B$-meson LCSR, in particular, the dependence on the heavy-mass scale $m_{B} \sim m_{b}$ at $q^{2}=0$ (at large energies of the final $P, V$ mesons).

Let us remind that the concept of $B$-meson DA's in its present form is only valid in the framework of HQET. In deriving the sum rules, we actually started from the formal $1 / m_{b}$ expansion (2) of the correlation function (1) and further used the HQET correlation function (3) expanding it in $B$-meson DA's. Hence, beyond the adopted approximation, there remain some unaccounted $1 / m_{b}$ corrections which contribute to the "systematical" uncertainty of our method. These corrections can be studied by expanding both the heavy-light current and the $B$-meson state in Eq. (1) beyond the leading-order in HQET.

As explained in Sect. 2,3, the relevant scale in the light-cone OPE for the HQET correlation function is the virtuality $P^{2}$ in the light-meson channel, or the corresponding Borel parameter $M^{2}$. This scale is chosen to be large with respect to $\Lambda_{Q C D}$ but is independent of $m_{b}$. In LCSR for the pion form factors 21, 24, 25 the higher-twist components of pion DA's, including the 3-particle (quarkantiquark-gluon) DA's, yield contributions that are normally suppressed by the inverse Borel scale. The absence of a well-defined twist in $B$ meson DA's makes the situation for the LCSR obtained here quite different. The contributions of the three-particle DA's do not reveal a general $1 / M^{2}$ suppression. Note however, that only the leading-order terms of the $x^{2}$-expansion for both quark-antiquark and quark-antiquark-gluon matrix elements (17) and (28) are taken into account. It is natural to expect that if one continues the light-cone expansion further, the $1 / M^{2}$ hierarchy will emerge in full scale.

The main source of $1 / m_{b}$ suppression in $B$-meson LCSR is related to the second large scale $m_{b} \omega$ present in the denominators of the correlation function (see Eq. (18)). In the sum rules (19)-(25) this scale manifests itself in the dependence of DA's on $\left(s / m_{B}\right)$, bounded by the duality interval $s / m_{B}<s_{0} / m_{B}$. The power of $1 / m_{b}$ suppression is entirely determined by the $\omega \rightarrow 0(\omega, \xi \rightarrow 0)$ "infrared" behavior of the $B$-meson two- (three-) particle DA's, contributing to the sum rule. This resembles the $1 / m_{b}$ expansion of the light-meson LCSR where the end-point behavior of the pion or $\rho$-meson DA's provides additional $1 / m_{b}$ suppression.

Expanding at $m_{b} \rightarrow \infty$ the LCSR (19)-(25), and adding the three-particle contributions given in the Appendix, one easily recovers the well-known relations 
[26] valid in the limit of the large light-meson energy $\left(E_{P, V} \sim m_{b} / 2\right)$ :

$$
\begin{aligned}
& f_{B P}^{+}(0)=\zeta, \quad f_{B P}^{T}(0)=\left(1+\frac{m_{P}}{m_{B}}\right) \zeta, \\
& V(0)=\left(1+\frac{m_{V}}{m_{B}}\right) \zeta_{\perp}, \quad A_{1}(0)=\frac{m_{B}}{m_{B}+m_{V}} \zeta_{\perp}, \\
& A_{2}(0)=\left(1+\frac{m_{V}}{m_{B}}\right)\left(\zeta_{\perp}-\frac{2 m_{V}}{m_{B}} \zeta_{\|}\right), \quad T_{1}(0)=\zeta_{\perp},
\end{aligned}
$$

For the first two universal form factors the following expressions in terms of $B$-meson DA's are obtained:

$$
\begin{aligned}
\zeta= & \frac{\hat{f}_{B}}{f_{P} m_{B}^{3 / 2}} e^{m_{P}^{2} / M^{2}} \int_{0}^{s_{0}^{P}} d s e^{-s / M^{2}} \phi_{-}^{B}(0) \\
\zeta_{\perp}= & \frac{\hat{f}_{B}}{2 f_{V} m_{V} m_{B}^{3 / 2}} e^{m_{V}^{2} / M^{2}} \int_{0}^{s_{0}^{V}} d s e^{-s / M^{2}}\left\{\left.s \frac{d \phi_{+}^{B}(\omega)}{d \omega}\right|_{\omega=0}\right. \\
& \left.-\int_{0}^{\infty} \frac{d \xi}{\xi} \Psi_{V}(0, \xi)\right\}
\end{aligned}
$$

where the $B$-meson decay constant is rescaled in a standard way: $f_{B}=\hat{f}_{B} / \sqrt{m_{b}}$. In the above, we neglected the light-quark masses $m_{1,2}$ but left $m_{P} \neq 0$ for generality. In deriving Eq. (57) we have also taken into account that the integral contributing to r.h.s.,

$$
\int_{0}^{\infty} \frac{d \xi}{\xi}\left(\Psi_{A}(0, \xi)+X_{A}(0, \xi)\right)=-\frac{1}{2} J(0)=0
$$

in our model. The third universal form factor $\zeta_{\|}$enters Eq. (55) for $A_{2}$ with an $O\left(1 / m_{B}\right)$ factor, hence, it cannot be cleanly separated from the other $1 / m_{b}$ corrections to the LCSR (24) for $A_{2}$. One has to obtain a separate sum rule for $A_{0}$, but we do not dwell on that here.

The $1 / m_{b}^{3 / 2}$ limit for all form factors, evident from Eqs. (56), (57), is consistent with the heavy-mass limit obtained from the light-meson LCSR. The only exception is the heavy-mass limit $f_{B \pi}^{+}+f_{B \pi}^{-} \sim O\left(1 / m_{b}^{5 / 2}\right)$, obtained from Eq. (20), and different from the $1 / m_{b}^{3 / 2}$-behavior predicted from LCSR with the pion DA's 27.

Our main observation is that the universal $B \rightarrow P$ form factor $\zeta$ does not receive contributions from the three-particle $B$ meson DA's, while for the $B \rightarrow V$ form factors the three-particle Fock components in the $B$-meson contribute at the leading power $O\left(1 / \mathrm{m}_{b}^{3 / 2}\right)$ with a universal term. This result agrees with the expectations of SCET discussed in [28. Also in the factorization formula for the form factor $\zeta$ derived in 29] the quark-antiquark-gluon DA's contribute at the leading order. 


\section{$6 \quad$ Numerical results}

To perform the numerical analysis of the new LCSR, we use the exponential model (43), (44) of $B$-meson DA's and adopt the interval [17]:

$$
\lambda_{B}(1 \mathrm{GeV})=460 \pm 110 \mathrm{MeV}
$$

for the inverse moment of $\phi_{+}^{B}$. The parameters $\lambda_{E}^{2}=\lambda_{H}^{2}$ are determined from Eq. (53), somewhat larger than in Eq. (38). In addition, having in mind the uncertainty of the model, we allow the parameters $\lambda_{E}=\lambda_{H}$ to vary within $\pm 50 \%$ at fixed $\lambda_{B}$, so that the constraints following from equations of motion remain valid. The $B$-meson decay constant $f_{B}=180 \pm 30 \mathrm{MeV}$ obtained from the two-point sum rule in $O\left(\alpha_{s}\right)$ is used, similar to [2]. This is consistent with the $O\left(\alpha_{s}\right)$ accuracy of $\lambda_{B}$. This matching of precisions is however not yet complete, in the absence of the $O\left(\alpha_{s}\right)$ corrections to LCSR.

The interval of Borel parameter adopted here, $M^{2}=1.0 \pm 0.5 \mathrm{GeV}^{2}$, is optimal for the two-point sum rules in the light-meson channels [6, 20, as well as for LCSR for the pion form factors [21, 24, 25]. Hence, the normalization scale of $\lambda_{B}$ is consistent with the average virtuality in the correlation function. The

\begin{tabular}{|c|l|c|}
\hline Meson & Decay constant [30] & Threshold parameter \\
\hline$\pi$ & $f_{\pi}=130.7 \pm 0.1 \mathrm{MeV}$ & $s_{0}^{\pi}=0.7 \mathrm{GeV}^{2}$ [6, 20] \\
\hline$K$ & $f_{K}=159.8 \pm 1.4 \pm 0.44 \mathrm{MeV}$ & $s_{0}^{K}=1.05 \mathrm{GeV}^{2}[\underline{31}$ \\
\hline$\rho$ & $f_{\rho}=209 \pm 2 \mathrm{MeV}$ & $s_{0}^{\rho}=1.6 \mathrm{GeV}^{2}[\underline{6}, \underline{20}]$ \\
\hline$K^{*}$ & $f_{K^{*}}=217 \pm 5 \mathrm{MeV}$ & $s_{0}^{K^{*}}=1.7 \mathrm{GeV}^{2}[32]$ \\
\hline
\end{tabular}

Table 2: Decay constants of light mesons and the threshold parameters extracted from the corresponding 2-point QCD sum rules.

input for various light-meson channels is listed in Table 2. As already mentioned, the duality-threshold parameter in each channel is fixed by adjusting the twopoint sum rule (taken with $O\left(\alpha_{s}\right)$ accuracy) to the experimentally measured decay constant. Note that the same values of $s_{0}^{\pi}$ and $s_{0}^{\rho}$ were used in LCSR for the pion electromagnetic [21, 24] and $\rho \pi \gamma, \pi \gamma \gamma^{*}$ [25] form factors, respectively.

For the channels with strange mesons we adopt $m_{s}(1 \mathrm{GeV})=130 \pm 10 \mathrm{MeV}$ which agrees, e.g., with the recent QCD sum rule estimates 33 .

To demonstrate the stability of the LCSR predictions with respect to the Borel parameter variation, as well as the role of three-particle corrections we plot the numerical results for the two representative form factors $f_{B \pi}^{+}(0)$ and $V^{B \rho}(0)$ in Fig. 4. The contribution of the three-particle DA's to the sum rule for $V^{B \rho}$ is substantially larger than the analogous contribution to the sum rule for $f_{B \pi}^{+}$; this observation is consistent with different $1 / m_{b}$ behavior of the three-particle corrections, as discussed in the previous section. Furthermore, to illustrate the sensitivity of $B$-meson LCSR to the value of the inverse moment $\lambda_{B}$, we plot in Fig. 5 our prediction for $f_{B \pi}(0)$ as a function of this input parameter.

The form factors at zero momentum transfer calculated with the input specified above are collected in second column of Table 3. To estimate the theoretical 


\begin{tabular}{|c|l|l|}
\hline form factor & this work & LCSR with light-meson DA's \\
\hline$f_{B \pi}^{+}(0)$ & $0.25 \pm 0.05$ & $0.258 \pm 0.031$ \\
\hline$f_{B K}^{+}(0)$ & $0.31 \pm 0.04$ & $0.301 \pm 0.041 \pm 0.008$ \\
\hline$f_{B \pi}^{T}(0)$ & $0.21 \pm 0.04$ & $0.253 \pm 0.028$ \\
\hline$f_{B K}^{T}(0)$ & $0.27 \pm 0.04$ & $0.321 \pm 0.037 \pm 0.009$ \\
\hline$V^{B \rho}(0)$ & $0.32 \pm 0.10$ & $0.323 \pm 0.029$ \\
\hline$V^{B K^{*}}(0)$ & $0.39 \pm 0.11$ & $0.411 \pm 0.033 \pm 0.031$ \\
\hline$A_{1}^{B \rho}(0)$ & $0.24 \pm 0.08$ & $0.242 \pm 0.024$ \\
\hline$A_{1}^{B K^{*}}(0)$ & $0.30 \pm 0.08$ & $0.292 \pm 0.028 \pm 0.023$ \\
\hline$A_{2}^{B \rho}(0)$ & $0.21 \pm 0.09$ & $0.221 \pm 0.023$ \\
\hline$A_{2}^{B K^{*}}(0)$ & $0.26 \pm 0.08$ & $0.259 \pm 0.027 \pm 0.022$ \\
\hline$T_{1}^{B \rho}(0)$ & $0.28 \pm 0.09$ & $0.267 \pm 0.021$ \\
\hline$T_{1}^{B K^{*}}(0)$ & $0.33 \pm 0.10$ & $0.333 \pm 0.028 \pm 0.024$ \\
\hline
\end{tabular}

Table 3: The $B \rightarrow \pi, K$ and $B \rightarrow \rho, K^{*}$ form factors calculated in this work, compared with the predictions of the light-meson LCSR obtained in [3] and [5], respectively. For the latter, the second uncertainty of the $B \rightarrow K\left(K^{*}\right)$ form factors is due to the first Gegenbauer moment in the kaon $\left(K^{*}\right) D A$, where $a_{1}^{K}(1 \mathrm{GeV})=0.05 \pm 0.03\left(a_{1}^{K^{*}}(1 \mathrm{GeV})=0.10 \pm 0.07\right)$ is taken. 

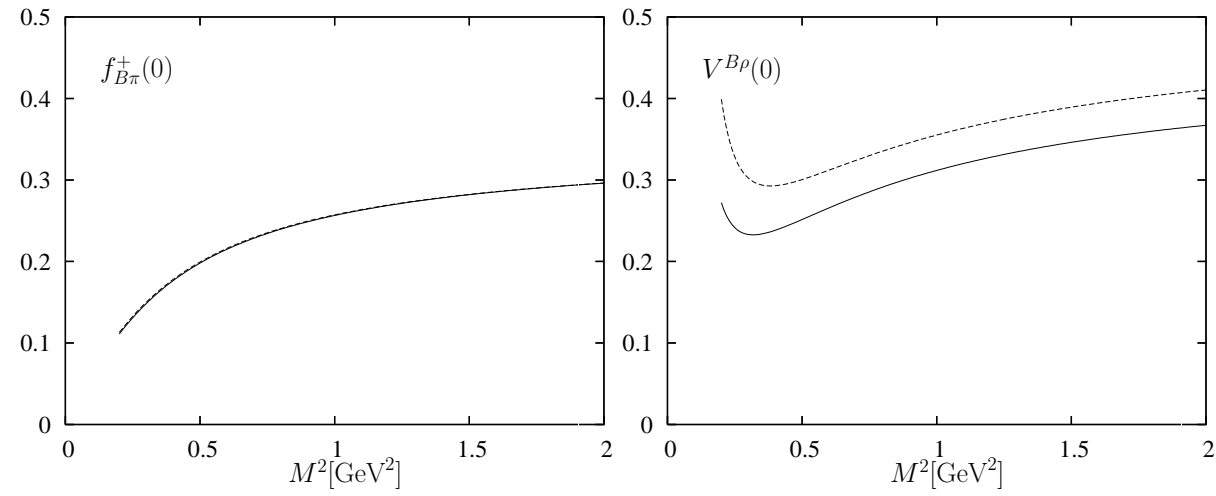

Figure 4: Dependence of the form factors $f_{B \pi}^{+}(0)$ (left) and $V^{B \rho}(0)$ (right) on the Borel parameter (solid lines). The contributions of two-particle DA's are shown by dashed lines, almost indistinguishable from the total result for $f_{B \pi}^{+}(0)$.

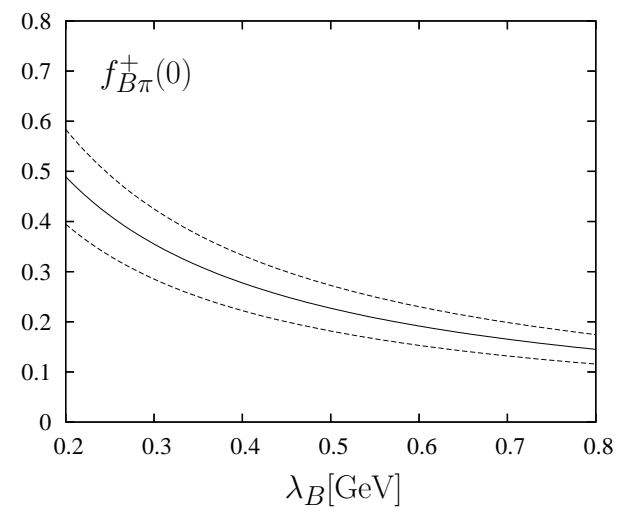

Figure 5: Dependence of the form factor $f_{B \pi}^{+}(0)$ on the inverse moment $\lambda_{B}$. The solid line corresponds to the central values of all other input parameters. Their variation yields the uncertainty interval shown with the dashed lines.

uncertainties, one usually adds linearly or in quadrature the uncertainties originating from separate variations of the input parameters. The intervals presented in Table 3 are obtained with a different procedure. The central value for each form factor is fitted to the set of LCSR predictions obtained by simultaneosly scanning all input parameters $\left(\lambda_{B}, \lambda_{E, H}^{2}, f_{B}, M^{2}, f_{P, V}\right.$ and $\left.m_{s}\right)$ within the adopted ranges. The errors attributed to the fitted values are the usual $1 \sigma$ deviations. The estimated uncertainties to a large extent originate from the interval of $\lambda_{B}$, hence they are larger for $B \rightarrow V$ form factors than for the $B \rightarrow P$ form factors, because the former (latter) mainly depend on $\phi_{+}^{B^{\prime}}(0) \sim \lambda_{B}^{-2}$ (on $\left.\phi_{-}^{B}(0) \sim \lambda_{B}^{-1}\right)$. Simultaneosly, the ratios of the form factors have much smaller uncertainties, in other words, the variations within the intervals presented in Table 3 are correlated. For example, the lower (upper) boundary of the interval for $T_{1}^{B \rho\left(B K^{*}\right)}(0)$ corresponds to the lower (upper) boundaries for $V^{B \rho\left(B K^{*}\right)}(0)$, $A_{1}^{B \rho,\left(B K^{*}\right)}(0)$ and $A_{2}^{B \rho\left(B K^{*}\right)}(0)$. 
In Table 3 the predictions of the $B$-meson LCSR are compared with the form factors obtained 3, 5, from the conventional light-meson LCSR. One has to keep in mind that the latter sum rules are more precise, because they include NLO corrections and are based on the well-developed twist expansion. Hence, the observed agreement between the predictions of two different methods is encouraging, possibly indicating that the unaccounted $O\left(\alpha_{s}\right)$ and power corrections to the new $B$-meson LCSR are not large.
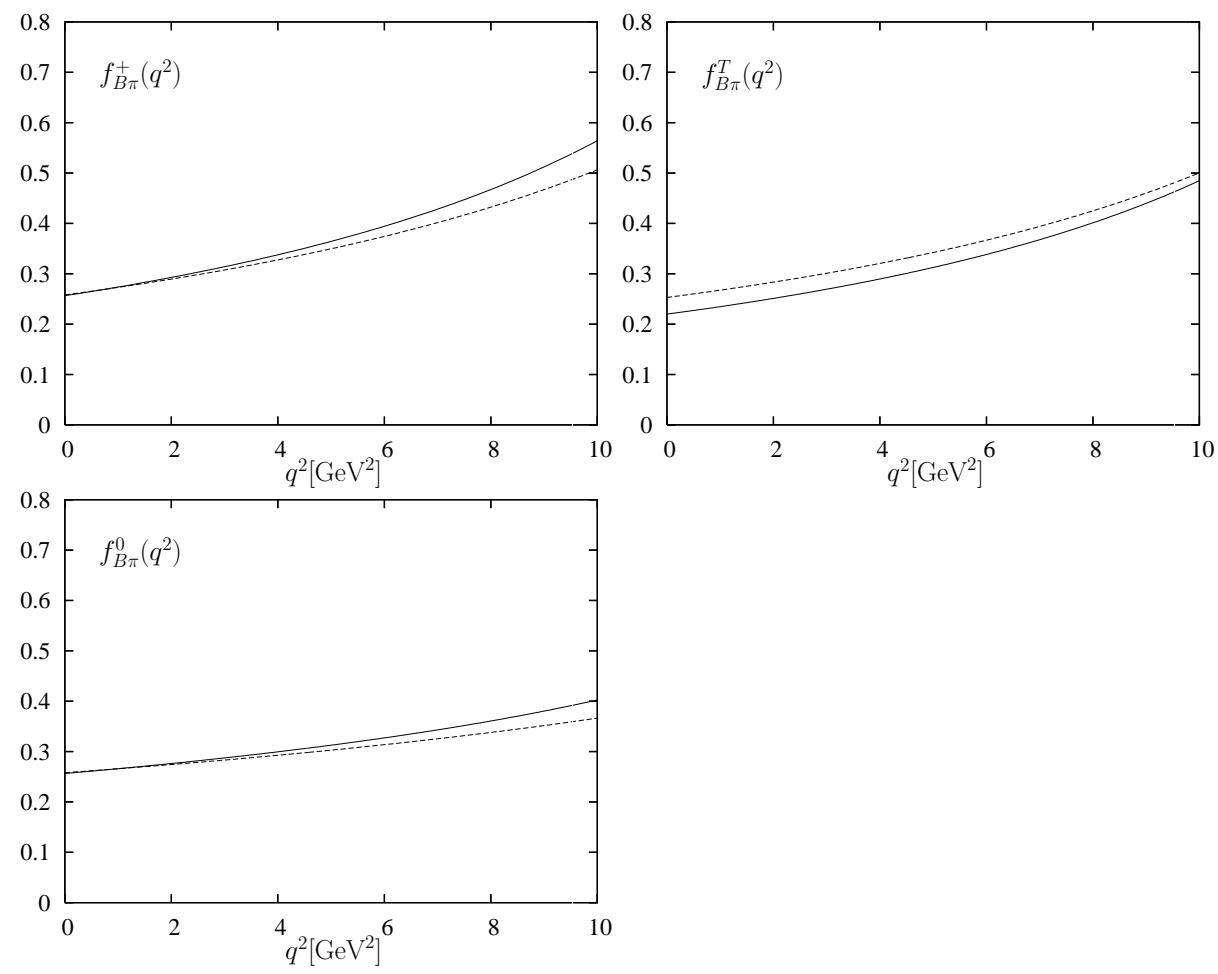

Figure 6: Dependence of the $B \rightarrow \pi$ form factors on the momentum transfer squared (solid lines) compared with the fits to the light-meson LCSR predictions from [3] (dashed lines). The theoretical uncertainties are not shown.

One more comment is in order. As already mentioned, in the $B$-meson and light-meson LCSR, quark-hadron duality is employed differently, in the lightmeson and $B$-meson channels, respectively. Hence, the difference between the predictions of two LCSR for one and the same form factor can be interpreted as a quantitative estimate of the "systematic" uncertainty caused by the duality ansatz. With the current accuracy of the $B$-meson LCSR we can only assess the upper limits for such uncertainties, by comparing the form factors in the second and third column of Table 3 . To substantiate these estimates, one has to enhance the accuracy of the $B$-meson LCSR, e.g., by including the perturbative corrections and narrowing the ranges of the major inputs parameters, such as $\lambda_{B}$.

The $B \rightarrow \pi, \rho$ form factors calculated at $q^{2} \neq 0$ are plotted in Figs. 6, (7, Note that to obtain the scalar form factor $f_{B \pi}^{0}\left(q^{2}\right)$ we have simply combined our predictions for $f_{B \pi}^{+}\left(q^{2}\right)$ and $f_{B \pi}^{+}\left(q^{2}\right)+f_{B \pi}^{-}\left(q^{2}\right)$. We evaluate the form factors 

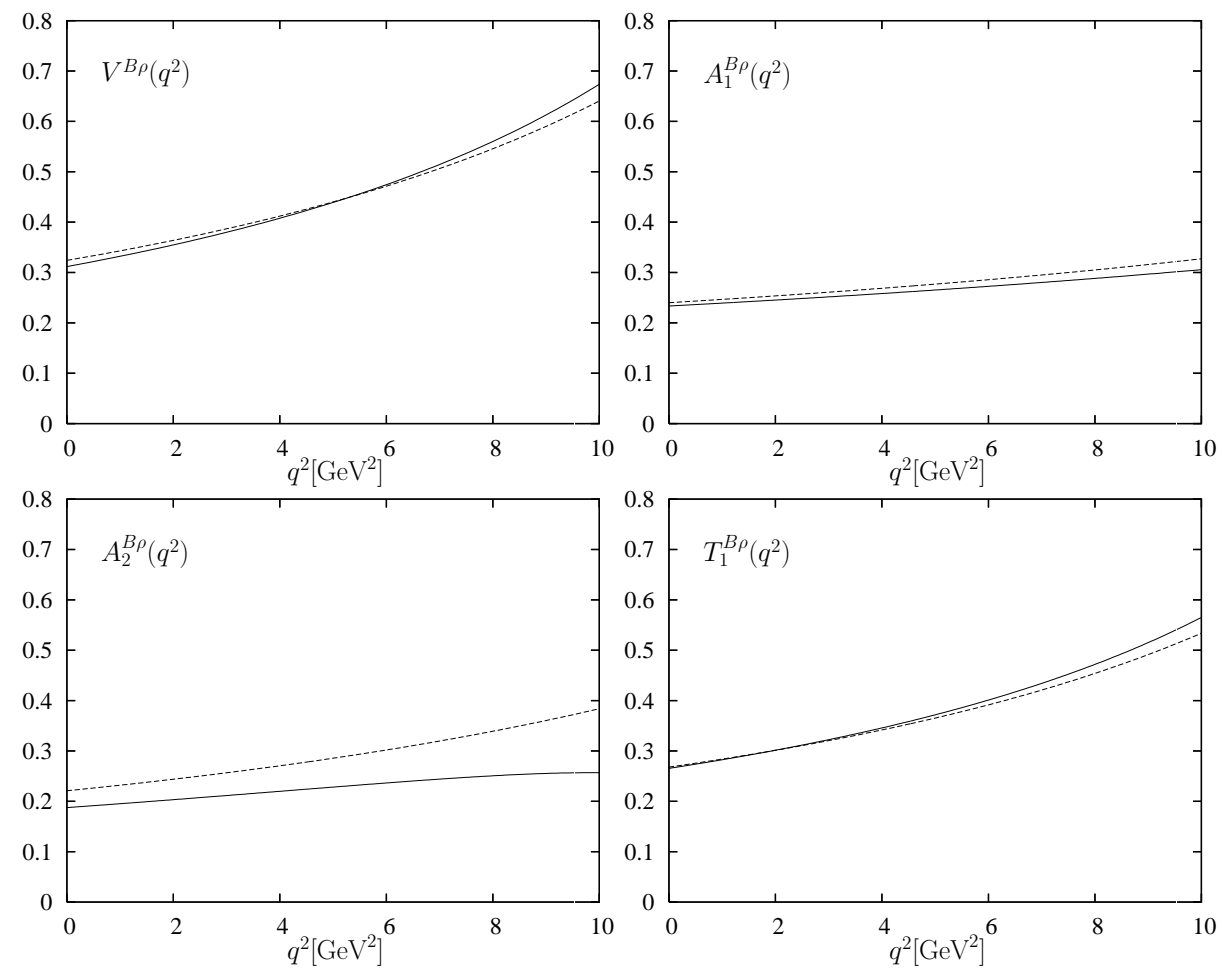

Figure 7: The same as in Fig. 6, but for the $B \rightarrow \rho$ form factors; the light-meson LCSR results are from [5].

at $0<q^{2}<10 \mathrm{GeV}^{2}$. The adopted range corresponds to the expected validity interval (7) where the upper boundary is taken at $P^{2} \sim M^{2}$. The light-meson LCSR are applicable 2, 3, at larger momentum transfers, up to $14-16 \mathrm{GeV}^{2}$. Note that the intervals presented in Table 3 include theoretical uncertainties, estimated as explained above, whereas the solid lines displayed in Figs. 6 7 have been evaluated at the central values of all parameters, hence, at $q^{2}=0$ these lines slightly deviate from the central points of the intervals. The estimated uncertainties of the form factors at $q^{2} \neq 0$, not shown here, are at the same level and have the same correlations as the uncertainties at $q^{2}=0$ discussed above.

Comparison with the results of the light-meson LCSR 3 , 5, reveals an agreement also at $q^{2} \neq 0$, as can be seen from Figs. 6, 7 taking into account also the uncertainties of both methods. To quantify the difference between the predictions of the two different types of LCSR at $q^{2} \neq 0$, we have fitted our results at $0<q^{2}<10 \mathrm{GeV}^{2}$ to the parametrization 34] used in [3, 5], e.g., for the $f_{B \pi}^{+}\left(q^{2}\right)$ form factor we employ:

$$
f_{B \pi}^{+}\left(q^{2}\right)=\frac{r_{1}}{1-q^{2} / m_{B^{*}}^{2}}+\frac{r_{2}}{1-q^{2} / m_{f i t}^{2}} .
$$

Since the adopted range of $q^{2}$ is rather narrow and the theoretical uncertainties are relatively large, it is difficult to fit all three parameters in Eq.(59) without 
producing an unphysically low mass $m_{f i t}$ of the second (effective) pole. The situation is improved if one adopts the value of $m_{f i t}=6.38 \mathrm{GeV}$ from [3] and fits only the residues of the two poles in (59). The result: $r_{1}=0.93$ and $r_{2}=-0.68$ has to be compared with $r_{1}=0.744$ and $r_{2}=-0.486$ obtained in $[3]$. The analogous fit of $B$-meson LCSR for the form factor $V^{B \rho}\left(q^{2}\right)$ yields (again at the fixed [5] mass $\left.m_{f i t}=6.19 \mathrm{GeV}\right)$ the residues: $r_{1}=1.10$ and $r_{2}=-0.80$, very close to $r_{1}=1.045$ and $r_{2}=-0.721$ obtained in [5]. A more detailed study of parameterizations at $q^{2} \neq 0$, including all $B \rightarrow P, V$ form factors, as well as the applications to heavy-light semileptonic and radiative decays, are subjects of a future study.

Furthermore, to illustrate the $S U(3)$-violation effects predicted from the $B$ meson LCSR, we have calculated the ratios:

$$
\begin{gathered}
\frac{f_{B K}^{+}(0)}{f_{B \pi}^{+}(0)}=1.27 \pm 0.07, \\
\frac{T_{1}^{B K^{*}}(0)}{T_{1}^{B \rho}(0)}=1.22 \pm 0.13 .
\end{gathered}
$$

Importantly, these ratios are much less dependent on the $B$-meson parameters, than the individual form factors. Our predictions are in agreement with the results obtained from the LCSR using DA's of strange and nonstrange light mesons; e.g., Eq. (60) can be compared with $f_{B K}^{+}(0) / f_{B \pi}^{+}(0)=1.36_{-.09}^{+.12}$ obtained in [31] and Eq. (61) with the most recent result [35]: $T_{1}^{B K^{*}}(0) / T_{1}^{B \rho}(0)=$ $1.17 \pm 0.09$. These are very important checks, because the new sum rules are independent of the $S U(3)$-violating Gegenbauer moments of the kaon and $K^{*}$.

In addition, returning to the $B \rightarrow \pi$ transition we predict the combination

$$
\frac{f_{B}}{f_{B \pi}^{+}(0) \lambda_{B}}=1.56 \pm 0.17
$$

which determines the coefficient of the hard-scattering contribution to the $B \rightarrow$ $\pi \pi$ amplitude in the QCD factorization approach (for a recent analysis, see e.g., [36]). Note, that within our method this ratio is practically independent of $\lambda_{B}$ and $f_{B}$ and is to a large extent determined by the parameters of the pion channel.

Summarizing, in this paper we have obtained a set of new QCD sum rules relating various $B \rightarrow P, V$ transition form factors to the universal light-cone DA's of B-meson. The contributions of the three-particle DA's to the new LCSR have been calculated. In addition, we studied the $B$-meson three-particle DA's, employing QCD sum rules in HQET, and have obtained a realistic exponential model of these DA's.

The correlation functions with an on-shell $B$ meson and a light-quark current allow many other applications to the heavy-light transitions, by simply changing the quantum numbers of the light-quark current. One does not need to install different light-meson DA's, and the two-point sum rules in the light-meson channels provide necessary information on the duality thresholds. With the interval of the inverse moment $\lambda_{B}$ from the QCD sum rules in HQET 9, 17, the numerical results obtained in this paper, including the $S U(3)$-violating ratios, provide a nontrivial check of the new method with respect to the light-meson LCSR. 
The new $B$-meson LCSR deserve further development. In this paper only the leading, zeroth order in $\alpha_{s}$ of the light-cone OPE has been taken into account. To complete the LCSR derivation at the NLO level, one has to calculate the QCD radiative corrections to the correlation function, involving the renormalization effects. In addition, further light-cone expansion of the two- and three-particle heavy-light matrix elements is desirable, in order to clarify the role of yet unaccounted $B$-meson DA's in generating $1 / m_{b}$ and/or $1 / M^{2}$ corrections to the sum rules. To obtain the necessary elements of these DA's, one can use the technique of HQET sum rules.

\section{Acknowledgements}

We are grateful to Vladimir Braun, Thorsten Feldmann and Andrei Grozin for useful discussions and comments. This work was supported by the Deutsche Forschungsgemeinschaft under the contract No. KH205/1-1 and by the German Minister of Research (BMBF, contract No. 05HT6PSA).

\section{Appendix}

Here the expressions for the LCSR at $q^{2} \neq 0$ and $m_{1} \equiv m \neq 0$, are presented $\left(m_{2}=0\right)$ :

- $B \rightarrow P$ form factors of the vector transition current

$$
\begin{gathered}
f_{B P}^{+}\left(q^{2}\right)=\frac{f_{B} m_{B}}{f_{P}}\left\{\int_{0}^{\sigma_{0}\left(q^{2}, s_{0}\right)} d \sigma \exp \left(\frac{-s\left(\sigma, q^{2}\right)+m_{P}^{2}}{M^{2}}\right)\right. \\
\times\left[\frac{\bar{\sigma}^{2} m_{B}^{2}}{\bar{\sigma}^{2} m_{B}^{2}+m^{2}-q^{2}} \phi_{-}^{B}\left(\sigma m_{B}\right)+\left(1-\frac{\bar{\sigma}^{2} m_{B}^{2}}{\bar{\sigma}^{2} m_{B}^{2}+m^{2}-q^{2}}\right) \phi_{+}^{B}\left(\sigma m_{B}\right)\right. \\
\left.\left.+\frac{2 \bar{\sigma}\left(m^{2}-q^{2}\right) m_{B}}{\left(\bar{\sigma}^{2} m_{B}^{2}+m^{2}-q^{2}\right)^{2}} \bar{\Phi}_{ \pm}^{B}\left(\sigma m_{B}\right)\right]+\Delta f_{B P}^{+}\left(q^{2}, s_{0}, M^{2}\right)\right\}, \quad(63) \\
f_{B P}^{+}\left(q^{2}\right)+f_{B P}^{-}\left(q^{2}\right)=\frac{f_{B} m_{B}}{f_{P}}\left\{\int_{0}^{\sigma_{0}\left(q^{2}, s_{0}\right)} d \sigma \exp \left(\frac{-s\left(\sigma, q^{2}\right)+m_{P}^{2}}{M^{2}}\right)\right. \\
\times\left[\frac{\left(m-2 \sigma \bar{\sigma} m_{B}\right) m_{B}}{\bar{\sigma}^{2} m_{B}^{2}+m^{2}-q^{2}} \phi_{-}^{B}\left(\sigma m_{B}\right)+\left(1-\frac{\sigma}{\bar{\sigma}}-\frac{\left(m-2 \sigma \bar{\sigma} m_{B}\right) m_{B}}{\bar{\sigma}^{2} m_{B}^{2}+m^{2}-q^{2}}\right) \phi_{+}^{B}\left(\sigma m_{B}\right)\right. \\
\left.-2 m_{B}\left(\frac{\bar{\sigma}\left(m-2 \sigma \bar{\sigma} m_{B}\right) m_{B}}{\left(\bar{\sigma}^{2} m_{B}^{2}+m^{2}-q^{2}\right)^{2}}+\frac{(\sigma-\bar{\sigma})}{\bar{\sigma}^{2} m_{B}^{2}+m^{2}-q^{2}}\right) \bar{\Phi}_{ \pm}^{B}\left(\sigma m_{B}\right)\right]
\end{gathered}
$$


- $B \rightarrow P$ form factor of the tensor current

$$
\begin{gathered}
f_{B P}^{T}\left(q^{2}\right)=\frac{f_{B}\left(m_{B}+m_{P}\right) m_{B}^{2}}{f_{P}\left(\left(m_{B}^{2}-m_{P}^{2}\right)-q^{2}\right)}\left\{\int_{0}^{\sigma_{0}\left(q^{2}, s_{0}\right)} d \sigma \exp \left(\frac{-s\left(\sigma, q^{2}\right)+m_{P}^{2}}{M^{2}}\right)\right. \\
\times\left[\frac{\bar{\sigma}^{2} m_{B}^{2}-m^{2}+(\sigma-\bar{\sigma}) q^{2}}{\bar{\sigma}^{2} m_{B}^{2}+m^{2}-q^{2}}\left(\phi_{-}^{B}\left(\sigma m_{B}\right)-\phi_{+}^{B}\left(\sigma m_{B}\right)\right)\right. \\
\left.+\frac{1}{m_{B}}\left(2 \frac{m^{2}\left(2 \bar{\sigma} m_{B}^{2}-q^{2}\right)+q^{2}\left(q^{2}-\bar{\sigma}(1+\sigma) m_{B}^{2}\right)}{\left(\bar{\sigma}^{2} m_{B}^{2}+m^{2}-q^{2}\right)^{2}}-\frac{1}{\bar{\sigma}}\right) \bar{\Phi}_{ \pm}^{B}\left(\sigma m_{B}\right)\right] \\
\left.+\Delta f_{B P}^{T}\left(q^{2}, s_{0}, M^{2}\right)\right\},
\end{gathered}
$$

- $B \rightarrow V$ form factor of the vector current

$$
\begin{gathered}
V^{B V}\left(q^{2}\right)=\frac{f_{B} m_{B}^{2}}{2 f_{V} m_{V}}\left(m_{B}+m_{V}\right)\left\{\int_{0}^{\sigma_{0}\left(q^{2}, s_{0}\right)} d \sigma \exp \left(\frac{-s\left(\sigma, q^{2}\right)+m_{V}^{2}}{M^{2}}\right)\right. \\
\times\left[\frac{m}{\bar{\sigma}^{2} m_{B}^{2}+m^{2}-q^{2}} \phi_{-}^{B}\left(\sigma m_{B}\right)+\left(\frac{1}{\bar{\sigma} m_{B}}-\frac{m}{\bar{\sigma}^{2} m_{B}^{2}+m^{2}-q^{2}}\right) \phi_{+}^{B}\left(\sigma m_{B}\right)\right. \\
\left.\left.-\frac{2 \bar{\sigma} m m_{B}}{\left(\bar{\sigma}^{2} m_{B}^{2}+m^{2}-q^{2}\right)^{2}} \bar{\Phi}_{ \pm}^{B}\left(\sigma m_{B}\right)\right]+\Delta V^{B V}\left(q^{2}, s_{0}, M^{2}\right)\right\}, \quad(66)
\end{gathered}
$$

- $B \rightarrow V$ form factors of the axial current

$$
\begin{gathered}
A_{1}^{B V}\left(q^{2}\right)=\frac{f_{B} m_{B}^{3}}{2 f_{V} m_{V}\left(m_{B}+m_{V}\right)}\left\{\int_{0}^{\sigma o\left(q^{2}, s_{0}\right)} d \sigma \exp \left(\frac{-s\left(\sigma, q^{2}\right)+m_{V}^{2}}{M^{2}}\right)\right. \\
\times\left[\frac { ( \overline { \sigma } m _ { B } + m ) ^ { 2 } - q ^ { 2 } } { m _ { B } ^ { 2 } \overline { \sigma } ^ { 2 } } \left\{\frac{\bar{\sigma} m m_{B}}{\bar{\sigma}^{2} m_{B}^{2}+m^{2}-q^{2}} \phi_{-}^{B}\left(\sigma m_{B}\right)\right.\right. \\
\left.\quad+\left(1-\frac{\bar{\sigma} m m_{B}}{\bar{\sigma}^{2} m_{B}^{2}+m^{2}-q^{2}}\right) \phi_{+}^{B}\left(\sigma m_{B}\right)\right\} \\
\left.\left.-4 \frac{\bar{\sigma} m^{2} m_{B}}{\left(\bar{\sigma}^{2} m_{B}^{2}+m^{2}-q^{2}\right)^{2}} \bar{\Phi}_{ \pm}^{B}\left(\sigma m_{B}\right)\right]+\Delta A_{1}^{B V}\left(q^{2}, s_{0}, M^{2}\right)\right\},
\end{gathered}
$$




$$
\begin{gathered}
A_{2}^{B V}\left(q^{2}\right)=\frac{f_{B} m_{B}}{2 f_{V} m_{V}}\left(m_{B}+m_{V}\right)\left\{\int_{0}^{\sigma_{0}\left(q^{2}, s_{0}\right)} d \sigma \exp \left(\frac{-s\left(\sigma, q^{2}\right)+m_{V}^{2}}{M^{2}}\right)\right. \\
\times\left[\frac{\left(m-2 \bar{\sigma} \sigma m_{B}\right) m_{B}}{\bar{\sigma}^{2} m_{B}^{2}+m^{2}-q^{2}} \phi_{-}^{B}\left(\sigma m_{B}\right)\right. \\
+\left(1-\frac{\sigma}{\bar{\sigma}}-\frac{\left(m-2 \bar{\sigma} \sigma m_{B}\right) m_{B}}{\bar{\sigma}^{2} m_{B}^{2}+m^{2}-q^{2}}\right) \phi_{+}^{B}\left(\sigma m_{B}\right) \\
\left.-2 m_{B}\left(\frac{\bar{\sigma}\left(m-2 \bar{\sigma} \sigma m_{B}\right) m_{B}}{\left(\bar{\sigma}^{2} m_{B}^{2}+m^{2}-q^{2}\right)^{2}}+\frac{(\sigma-\bar{\sigma})}{\bar{\sigma}^{2} m_{B}^{2}+m^{2}-q^{2}}\right) \bar{\Phi}_{ \pm}^{B}\left(\sigma m_{B}\right)\right] \\
\left.+\Delta A_{2}^{B V}\left(q^{2}, s_{0}, M^{2}\right)\right\},
\end{gathered}
$$

- $B \rightarrow V$ form factor of the tensor current

$$
\begin{gathered}
T_{1}^{B V}\left(q^{2}\right)=\frac{f_{B} m_{B}^{2}}{2 f_{V} m_{V}}\left\{\int_{0}^{\sigma_{0}\left(q^{2}, s_{0}\right)} d \sigma \exp \left(\frac{-s\left(\sigma, q^{2}\right)+m_{V}^{2}}{M^{2}}\right)\right. \\
\quad\left[( 1 + \frac { m } { \overline { \sigma } m _ { B } } ) \left\{\frac{\bar{\sigma} m m_{B}}{\bar{\sigma}^{2} m_{B}^{2}+m^{2}-q^{2}} \phi_{-}^{B}\left(\sigma m_{B}\right)\right.\right. \\
\left.\quad+\left(1-\frac{\bar{\sigma} m m_{B}}{\bar{\sigma}^{2} m_{B}^{2}+m^{2}-q^{2}}\right) \phi_{+}^{B}\left(\sigma m_{B}\right)\right\} \\
\left.+\frac{m}{\bar{\sigma}^{2} m_{B}^{2}+m^{2}-q^{2}}\left(1-\frac{2 \bar{\sigma} m_{B}\left(\bar{\sigma} m_{B}+m\right)}{\bar{\sigma}^{2} m_{B}^{2}+m^{2}-q^{2}}\right) \bar{\Phi}_{ \pm}^{B}\left(\sigma m_{B}\right)\right] \\
\left.+\Delta T_{1}^{B V}\left(q^{2}, s_{0}, M^{2}\right)\right\},
\end{gathered}
$$

where, in order to compactify the above expressions we use the dimensionless integration variable $\sigma=\omega / m_{B}$ and the following notations: $\bar{\sigma}=1-\sigma$,

$$
\begin{gathered}
s\left(\sigma, q^{2}\right)=\sigma m_{B}^{2}+\frac{m^{2}-\sigma q^{2}}{\bar{\sigma}}, \\
\sigma_{0}\left(q^{2}, s_{0}\right)=\frac{m_{B}^{2}-q^{2}+s_{0}-\sqrt{4\left(m^{2}-s_{0}\right) m_{B}^{2}+\left(m_{B}^{2}-q^{2}+s_{0}\right)^{2}}}{2 m_{B}^{2}},
\end{gathered}
$$

so that at $m=0$ and $q^{2}=0, s(\sigma, 0)=\sigma m_{B}^{2}$ and $\sigma_{0}\left(0, s_{0}\right)=s_{0} / m_{B}^{2}$.

In the above $\Delta f_{B P}^{+}, \Delta f_{B P}^{ \pm}, \Delta f_{B P}^{T}, \Delta V^{B V}, \Delta A_{1}^{B V}, \Delta A_{2}^{B V}$, and $\Delta T_{1}^{B V}$ denote the contribuitons of the $B$-meson three-particle DA's. We obtain the following 
generic formula for these correction ( $\Delta F=\Delta f_{B P}^{+}, \Delta f_{B P}^{ \pm}$, etc.):

$$
\begin{aligned}
& \Delta F\left(q^{2}, s_{0}, M^{2}\right)= \int_{0}^{\sigma_{0}\left(q^{2}, s_{0}\right)} d \sigma \exp \left(\frac{-s\left(\sigma, q^{2}\right)+m_{P(V)}^{2}}{M^{2}}\right) \\
& \times\left(-I_{1}^{(F)}(\sigma)+\frac{I_{2}^{(F)}(\sigma)}{M^{2}}-\frac{I_{3}^{(F)}(\sigma)}{2 M^{4}}\right) \\
&+\frac{e^{\left(-s_{0}+m_{P(V)}^{2}\right) / M^{2}}}{m_{B}^{2}}\left\{\eta ( \sigma ) \left[I_{2}^{(F)}(\sigma)\right.\right. \\
&\left.\left.-\frac{1}{2}\left(\frac{1}{M^{2}}+\frac{1}{m_{B}^{2}} \frac{d \eta(\sigma)}{d \sigma}\right) I_{3}^{(F)}(\sigma)-\frac{\eta(\sigma)}{2 m_{B}^{2}} \frac{d I_{3}^{(F)}(\sigma)}{d \sigma}\right]\right\}\left.\right|_{\sigma=\sigma_{0}},
\end{aligned}
$$

where

$$
\eta(\sigma)=\left(1+\frac{m^{2}-q^{2}}{\bar{\sigma}^{2} m_{B}^{2}}\right)^{-1},
$$

and the integrals over the three-particle DA's multiplying the inverse powers of the Borel parameter $1 / M^{2(n-1)}$ with $n=1,2,3$ are defined as:

$$
\begin{aligned}
& I_{n}^{(F)}(\sigma)=\frac{1}{\bar{\sigma}^{n}} \int_{0}^{\sigma m_{B}} d \omega \int_{\sigma m_{B}-\omega}^{\infty} \frac{d \xi}{\xi}\left[C_{n}^{(F, \Psi A)}\left(\sigma, u, q^{2}\right) \Psi_{A}^{B}(\omega, \xi)\right. \\
& \quad+C_{n}^{(F, \Psi V)}\left(\sigma, u, q^{2}\right) \Psi_{V}^{B}(\omega, \xi) \\
& \left.+C_{n}^{(F, X A)}\left(\sigma, u, q^{2}\right) \bar{X}_{A}^{B}(\omega, \xi)+C_{n}^{(F, Y A)}\left(\sigma, u, q^{2}\right) \bar{Y}_{A}^{B}(\omega, \xi)\right]\left.\right|_{u=\left(\sigma m_{B}-\omega\right) / \xi}
\end{aligned}
$$

where:

$$
\bar{X}_{A}^{B}(\omega, \xi)=\int_{0}^{\omega} d \tau X_{A}^{B}(\tau, \xi), \quad \bar{Y}_{A}^{B}(\omega, \xi)=\int_{0}^{\omega} d \tau Y_{A}^{B}(\tau, \xi)
$$


The nonvanishing coefficients entering Eq. (72) are:

$$
\begin{aligned}
& C_{2}^{\left(f_{B P}^{+}, \Psi A\right)}=\left(-\frac{2 m}{m_{B}}+\bar{\sigma}-2 \bar{\sigma} u\right), \quad C_{2}^{\left(f_{B P}^{+}, \Psi V\right)}=\left(\frac{2 m}{m_{B}}+\bar{\sigma}\right), \\
& C_{2}^{\left(f_{B P}^{+}, X A\right)}=\frac{1-2 u}{m_{B}}, C_{3}^{\left(f_{B P}^{+}, X A\right)}=2 \frac{\left(m^{2}+q^{2}-m_{B}^{2} \bar{\sigma}^{2}\right)(2 u-1)}{m_{B}}, \\
& C_{3}^{\left(f_{B P}^{+}, Y A\right)}=4\left(2 m+m_{B} \bar{\sigma}(2 u-1)\right) \bar{\sigma}, \\
& C_{2}^{\left(f_{B P}^{ \pm}, \Psi A\right)}=1-\frac{4 m}{m_{B}}+\bar{\sigma}(2-4 u)+2 u, \quad C_{2}^{\left(f_{B P}^{ \pm}, \Psi V\right)}=1+\frac{4 m}{m_{B}}+2 \bar{\sigma}-4 u, \\
& C_{2}^{\left(f_{B P}^{ \pm}, X A\right)}=-\frac{2(\bar{\sigma}-1)(2 u-1)}{m_{B} \bar{\sigma}}, \\
& C_{3}^{\left(f_{B P}^{ \pm}, X A\right)}=-\frac{2}{m_{B} \bar{\sigma}}\left(-(2 \bar{\sigma}+1)(2 u-1) m^{2}+2 m m_{B} \bar{\sigma}+\right. \\
& \left.(2 \bar{\sigma}-1)\left(m_{B}^{2} \bar{\sigma}^{2}-q^{2}\right)(2 u-1)\right) \\
& C_{3}^{\left(f_{B P}^{ \pm}, Y A\right)}=4\left(m(4 \bar{\sigma}-1)+2 m_{B}(\bar{\sigma}-1) \bar{\sigma}(2 u-1)\right), \\
& C_{1}^{\left(f_{B P}^{T}, \Psi A\right)}=\frac{2 u}{m_{B}^{2} \bar{\sigma}}, \quad C_{2}^{\left(f_{B P}^{T}, \Psi A\right)}=-\frac{2\left(-m^{2}+m_{B}^{2} \bar{\sigma}^{2}+q^{2}(1-2 \bar{\sigma})\right) u}{m_{B}^{2} \bar{\sigma}}, \\
& C_{1}^{\left(f_{B P}^{T}, \Psi V\right)}=-\frac{2 u}{m_{B}^{2} \bar{\sigma}}, \quad C_{2}^{\left(f_{B P}^{T}, \Psi V\right)}=-\frac{2\left(m^{2}-m_{B}^{2} \bar{\sigma}^{2}+q^{2}(2 \bar{\sigma}-1)\right) u}{m_{B}^{2} \bar{\sigma}}, \\
& C_{2}^{\left(f_{B P}^{T}, X A\right)}=-\frac{4 m}{m_{B}^{2} \bar{\sigma}}, C_{3}^{\left(f_{B P}^{T}, X A\right)}=-\frac{4 m\left(m^{2}-m_{B}^{2} \bar{\sigma}^{2}+q^{2}(2 \bar{\sigma}-1)\right)}{m_{B}^{2} \bar{\sigma}}, \\
& C_{2}^{\left(f_{B P}^{T}, Y A\right)}=-4 \frac{(2 u-1)}{m_{B}}, \\
& C_{3}^{\left(f_{B P}^{T}, Y A\right)}=4 \frac{\left(-m^{2}+m_{B}^{2} \bar{\sigma}^{2}+q^{2}(1-2 \bar{\sigma})\right)(2 u-1)}{m_{B}}, \\
& C_{2}^{\left(V^{B V}, \Psi A\right)}=\frac{2 u-1}{m_{B}}, \quad C_{2}^{\left(V^{B V}, \Psi V\right)}=-\frac{1}{m_{B}}, \quad C_{2}^{\left(V^{B V}, X A\right)}=-2 \frac{(2 u-1)}{m_{B}^{2} \bar{\sigma}}, \\
& C_{3}^{\left(V^{B V}, X A\right)}=-\frac{2}{m_{B}^{2} \bar{\sigma}}\left((2 u-1) m^{2}-2 m_{B} \bar{\sigma} m+\left(m_{B}^{2} \bar{\sigma}^{2}-q^{2}\right)(2 u-1)\right), \\
& C_{3}^{\left(V^{B V}, Y A\right)}=-4 \frac{m}{m_{B}},
\end{aligned}
$$




$$
\begin{aligned}
& C_{1}^{\left(A_{1}^{B V}, \Psi A\right)}=\frac{2 u-1}{m_{B}^{2} \bar{\sigma}}, \\
& C_{2}^{\left(A_{1}^{B V}, \Psi A\right)}=\frac{m^{2}(2 u-1)+2 m m_{B} \bar{\sigma}-\left(q^{2}-m_{B}^{2} \bar{\sigma}^{2}\right)(2 u-1)}{m_{B}^{2} \bar{\sigma}}, \\
& C_{1}^{\left(A_{1}^{B V}, \Psi V\right)}=-\frac{1}{m_{B}^{2} \bar{\sigma}}, \quad C_{2}^{\left(A_{1}^{B V}, \Psi V\right)}=-\frac{\left(m^{2}+2 m m_{B} \bar{\sigma}-q^{2}+m_{B}^{2} \bar{\sigma}^{2}\right)}{m_{B}^{2} \bar{\sigma}}, \\
& C_{1}^{\left(A_{1}^{B V}, X A\right)}=-2 \frac{(2 u-1)}{m_{B}^{3} \bar{\sigma}^{2}}, \\
& C_{2}^{\left(A_{1}^{B V}, X A\right)}=-\frac{2\left(2 m^{2}-2 q^{2}+m_{B}^{2} \bar{\sigma}^{2}\right)(2 u-1)}{m_{B}^{3} \bar{\sigma}^{2}}, \\
& C_{3}^{\left(A_{1}^{B V}, X A\right)}=-\frac{2\left(m^{4}-2 m^{2}\left(q^{2}+m_{B}^{2} \bar{\sigma}^{2}\right)+\left(q^{2}-m_{B}^{2} \bar{\sigma}^{2}\right)^{2}\right)(2 u-1)}{m_{B}^{3} \bar{\sigma}^{2}}, \\
& C_{2}^{\left(A_{1}^{B V}, Y A\right)}=-\frac{4\left(m+m_{B} \bar{\sigma}(1-2 u)\right)}{m_{B}^{2} \bar{\sigma}}, \\
& C_{3}^{\left(A_{1}^{B V}, Y A\right)}=-\frac{4 m\left(m^{2}+2 m_{B} \bar{\sigma}(2 u-1) m-q^{2}+m_{B}^{2} \bar{\sigma}^{2}\right)}{m_{B}^{2} \bar{\sigma}}, \\
& C_{2}^{\left(A_{2}^{B V}, \Psi A\right)}=-\left(3-\frac{4 m}{m_{B}}-2 u+\bar{\sigma}(4 u-2)\right), \\
& C_{2}^{\left(A_{2}^{B V}, \Psi V\right)}=-\left(3+\frac{4 m}{m_{B}}-2 \bar{\sigma}-4 u\right), \\
& C_{2}^{\left(A_{2}^{B V}, X A\right)}=-\frac{2(\bar{\sigma}-1)(2 u-1)}{m_{B} \bar{\sigma}}, \\
& C_{3}^{\left(A_{2}^{B V}, X A\right)}=-\frac{2}{m_{B} \bar{\sigma}}\left(-(2 \bar{\sigma}+1)(2 u-1) m^{2}+2 m m_{B} \bar{\sigma}\right. \\
& \left.+(2 \bar{\sigma}-1)\left(m_{B}^{2} \bar{\sigma}^{2}-q^{2}\right)(2 u-1)\right), \\
& C_{3}^{\left(A_{2}^{B V}, Y A\right)}=4\left(m(3-4 \bar{\sigma})+2 m_{B}(\bar{\sigma}-1) \bar{\sigma}(2 u-1)\right), \\
& C_{2}^{\left(T_{1}^{B V}, \Psi A\right)}=\frac{\left(m+m_{B} \bar{\sigma}(2 u-1)\right)}{m_{B}}, \quad C_{2}^{\left(T_{1}^{B V}, \Psi V\right)}=-\frac{\left(m+m_{B} \bar{\sigma}\right)}{m_{B}}, \\
& C_{2}^{\left(T_{1}^{B V}, X A\right)}=\frac{1-2 u}{m_{B}}-\frac{2 m}{m_{B}^{2} \bar{\sigma}}, \\
& C_{3}^{\left(T_{1}^{B V}, X A\right)}=-\frac{2}{m_{B}^{2} \bar{\sigma}}\left(m^{3}+m^{2} m_{B} \bar{\sigma}(1-2 u)-m\left(q^{2}+m_{B}^{2} \bar{\sigma}^{2}\right)\right. \\
& \left.+m_{B} \bar{\sigma}\left(m_{B}^{2} \bar{\sigma}^{2}-q^{2}\right)(2 u-1)\right), \\
& C_{2}^{\left(T_{1}^{B V}, Y A\right)}=2 \frac{(2 u-1)}{m_{B}}, \quad C_{3}^{\left(T_{1}^{B V}, Y A\right)}=-4 \frac{m\left(m_{B} \bar{\sigma}+m(2 u-1)\right)}{m_{B}} .
\end{aligned}
$$




\section{References}

[1] I. I. Balitsky, V. M. Braun and A. V. Kolesnichenko, Nucl. Phys. B312 (1989) 509; V. M. Braun and I. E. Halperin, Z. Phys. C 44 (1989) 157; V. L. Chernyak and I. R. Zhitnitsky, Nucl. Phys. B345 (1990) 137.

[2] V. M. Belyaev, A. Khodjamirian and R. Rückl, Z. Phys. C 60 (1993) 349; V. M. Belyaev, V. M. Braun, A. Khodjamirian and R. Rückl, Phys. Rev. D 51 (1995) 6177; A. Khodjamirian, R. Rückl, S. Weinzierl and O. I. Yakovlev, Phys. Lett. B 410 (1997) 275; E. Bagan, P. Ball and V. M. Braun, Phys. Lett. B 417 (1998) 154; P. Ball, JHEP 9809, 005 (1998). P. Ball and R. Zwicky, JHEP 0110 (2001) 019.

[3] P. Ball and R. Zwicky, Phys. Rev. D 71 (2005) 014015.

[4] A. Ali, V. M. Braun and H. Simma, Z. Phys. C 63 (1994) 437; P. Ball and V. M. Braun, Phys. Rev. D 55 (1997) 5561; Phys. Rev. D 58 (1998) 094016 .

[5] P. Ball and R. Zwicky, Phys. Rev. D 71 (2005) 014029.

[6] M. A. Shifman, A. I. Vainshtein and V. I. Zakharov, Nucl. Phys. B 147, 385,448 (1979).

[7] A. Khodjamirian, T. Mannel and N. Offen, Phys. Lett. B 620 (2005) 52.

[8] F. De Fazio, T. Feldmann and T. Hurth, Nucl. Phys. B 733, 1 (2006).

[9] A. G. Grozin and M. Neubert, Phys. Rev. D 55 (1997) 272.

[10] A. Szczepaniak, E. M. Henley and S. J. Brodsky, Phys. Lett. B 243 (1990) 287.

[11] A. G. Grozin, Int. J. Mod. Phys. A 20, 7451 (2005).

[12] M. Beneke and T. Feldmann, Nucl. Phys. B 592 (2001) 3.

[13] G. P. Korchemsky, D. Pirjol and T. M. Yan, Phys. Rev. D 61 (2000) 114510; S. Descotes-Genon and C. T. Sachrajda, Nucl. Phys. B 650 (2003) 356; E. Lunghi, D. Pirjol and D. Wyler, Nucl. Phys. B 649 (2003) 349;

S. W. Bosch, R. J. Hill, B. O. Lange and M. Neubert, Phys. Rev. D 67 (2003) 094014.

[14] M. Beneke, G. Buchalla, M. Neubert and C. T. Sachrajda, Nucl. Phys. B 591 (2000) 313; Nucl. Phys. B 606 (2001) 245.

[15] M. Beneke, T. Feldmann and D. Seidel, Nucl. Phys. B 612 (2001) 25;

S. W. Bosch and G. Buchalla, Nucl. Phys. B 621 (2002) 459.

[16] H. Kawamura, J. Kodaira, C. F. Qiao and K. Tanaka, Phys. Lett. B 523, 111 (2001) [Erratum-ibid. B 536, 344 (2002)].

[17] V. M. Braun, D. Y. Ivanov and G. P. Korchemsky, Phys. Rev. D 69 (2004) 034014 .

[18] S. J. Lee and M. Neubert, Phys. Rev. D 72, 094028 (2005). 
[19] B. O. Lange and M. Neubert, Phys. Rev. Lett. 91 (2003) 102001.

[20] P. Colangelo and A. Khodjamirian, arXiv:hep-ph/0010175.

[21] V. M. Braun, A. Khodjamirian and M. Maul, Phys. Rev. D 61 (2000) 073004

[22] I. I. Balitsky and V. M. Braun, Nucl. Phys. B 311 (1989) 541.

[23] B. Geyer and O. Witzel, Phys. Rev. D 72 (2005) 034023.

[24] V. M. Braun and I. E. Halperin, Phys. Lett. B 328 (1994) 457; J. Bijnens and A. Khodjamirian, Eur. Phys. J. C 26 (2002) 67.

[25] A. Khodjamirian, Eur. Phys. J. C 6 (1999) 477.

[26] J. Charles, A. Le Yaouanc, L. Oliver, O. Pene and J. C. Raynal, Phys. Rev. D 60 (1999) 014001.

[27] A. Khodjamirian, R. Rückl and C. W. Winhart, Phys. Rev. D 58 (1998) 054013 .

[28] B. O. Lange, Eur. Phys. J. C 33 (2004) S324 [Nucl. Phys. Proc. Suppl. 133 (2004) 174].

[29] A. V. Manohar and I. W. Stewart, arXiv:hep-ph/0605001.

[30] W. M. Yao et al. [Particle Data Group], J. Phys. G 33 (2006) 1.

[31] A. Khodjamirian, T. Mannel and M. Melcher, Phys. Rev. D 68 (2003) 114007; Phys. Rev. D 70 (2004) 094002.

[32] P. Ball and R. Zwicky, Phys. Lett. B 633 (2006) 289.

[33] K. G. Chetyrkin and A. Khodjamirian, Eur. Phys. J. C 46 (2006) 721;

M. Jamin, J. A. Oller and A. Pich, Phys. Rev. D 74 (2006) 074009.

[34] D. Becirevic and A. B. Kaidalov, Phys. Lett. B 478 (2000) 417.

[35] P. Ball and R. Zwicky, JHEP 0604 (2006) 046.

[36] M. Beneke and S. Jäger, Nucl. Phys. B 751, 160 (2006). 